\title{
POINCARÉ THEORY FOR DECOMPOSABLE COFRONTIERS
}

\author{
T. Jäger* and A. Koropecki ${ }^{\dagger}$
}

\begin{abstract}
We extend Poincaré's theory of orientation-preserving homeomorphisms from the circle to circloids with decomposable boundary. As special cases, this includes both decomposable cofrontiers and decomposable cobasin boundaries. More precisely, we show that if the rotation number on an invariant circloid $A$ of a surface homeomorphism is irrational and the boundary of $A$ is decomposable, then the dynamics are monotonically semiconjugate to the respective irrational rotation. This complements classical results by Barge and Gillette on the equivalence between rational rotation numbers and the existence of periodic orbits and yields a direct analogue to the Poincaré Classification Theorem for circle homeomorphisms. Moreover, we show that the semiconjugacy can be obtained as the composition of a monotone circle map with a 'universal factor map', only depending on the topological structure of the circloid. This implies, in particular, that the monotone semiconjugacy is unique up to post-composition with a rotation.

If, in addition, $A$ is a minimal set, then the semiconjugacy is almost one-to-one if and only if there exists a biaccessible point. In this case, the dynamics on $A$ are almost automorphic. Conversely, we use the Anosov-Katok method to build a $C^{\infty}$-example where all fibres of the semiconjugacy are non-trivial.
\end{abstract}

\section{Introduction}

Given an orientation-preserving circle homeomorphism $\varphi$, the Poincaré Classification Theorem states that the rotation number $\rho(\varphi)$ is well-defined and determines the qualitative dynamical behaviour of $\varphi$, in the sense that $\rho(\varphi)$ is rational if and only if $\varphi$ has a periodic orbit and irrational if and only if $\varphi$ is monotonically semiconjugate to the respective irrational rotation. This result provides the basis for a rather complete understanding of invertible dynamics on the circle. At the same time the study of cofrontiers, circloids and other classes of circle-like continua, like basin boundaries, co-basin boundaries or pseudocircles, has a long history in plane topology and continuum theory, going back to Kuratowski, Cartwright, Littlewood, Bing and others Kur28, CL51a, Bin51. Recently the topic has gained further momentum, since invariant circloids play a crucial role in surface dynamics. It is therefore a natural question to ask whether an analogue to Poincaré's classical result holds for these more general continua. In this article, we extend the Poincaré Classification to circloids with decomposable boundary ${ }^{1}$ In order to define this notion, we let $\mathbb{T}^{1}=\mathbb{R} / \mathbb{Z}$ and $\mathbb{A}=\mathbb{T}^{1} \times \mathbb{R}$ and call a continuum $A \subseteq \mathbb{A}$ an essential annular continuum if $\mathbb{A} \backslash A$ consists of exactly two connected components, both of which are homeomorphic to $\mathbb{A}$. Further, $A$ is called an essential circloid if it does not contain any strictly smaller essential annular continuum as a subset, and a cofrontier if it is a circloid with empty interior. We refer to Section 2 for further explanations and details.

In the context of the dynamics of surface homeomorphisms, circloids may appear in various situations. For instance, they separate adjacent invariant topological disks or annular domains BG91, BGM93, Wal91, KY94, FLC03, and any periodic point free continuum of a non-wandering surface homeomorphism is an annular continuum Kor10 that can further be decomposed into a dense union of invariant circloids and transitive annuli Jäg10. On the two-torus, the existence of invariant circloids can often be deduced from information on the rotation set Jäg09a, Dav, GKT14, and invariant "foliations"

\footnotetext{
*Department of Mathematics, TU Dresden, Germany. Email: Tobias.Dertel-Jaeger@tu-dresden.de

${ }^{\dagger}$ Universidade Federal Fluminense, Niterói, Brasil. Email: ak@id.uff.br

${ }^{1}$ We recall that a continuum is called decomposable if it can be written as the union of two non-empty proper subcontinua.
} 
consisting of circloids play an important role for the problem of linearisation Jäg09b. The respective results in topological dynamics have further applications in the theory of $C^{r}$-generic diffeomorphisms [FLC03, KN10, KLCN15. It is thus of vital interest to understand the interplay between the topological structure and the possible dynamical behaviour on such continua. However, while the relation between rational rotation numbers and periodic orbits is quite well-understood [CL51a, BG91, KLCN15, the more intricate question of irrational rotation factors has been left completely open so far.

The problem is complicated by the fact that the rotation number on invariant circloids is not necessarily unique. Non-degenerate rotation intervals have been shown to occur on the Birkhoff attractor [LC88] and, more recently, the pseudocircle [BO]. Such examples can be excluded by adding a mild recurrence assumption [KLCN15], but even in the case of a unique rotation number a semiconjugacy does not have to exist. This was shown by Handel [Han82] and Herman Her86], who realised the pseudocircle as a minimal set of a smooth surface diffeomorphism. In these examples, the rotation number is irrational, but the dynamics are not semiconjugate to the corresponding rotation. While the pseudocircle is the paradigm example of a circle-like continuum with highly intricate topological structure, a modification of the construction can be used to produce a variety of more 'regular' indecomposable continua with the same behaviour. Hence, decomposability of the circloid presents itself as the obvious minimal requirement for a possible analogue to the Poincaré classification. As the following result shows, it turns out to be sufficient as well. Recall that a monotone map is one with connected fibers.

Theorem 1.1. Suppose $\varphi: \mathbb{A} \rightarrow \mathbb{A}$ is a homeomorphism homotopic to the identity with an essential $\varphi$-invariant circloid $A$ with decomposable boundary. Then every point of $A$ has a well-defined rotation number $\rho \in \mathbb{T}^{1}$ which is independent of the point, and

- $\rho$ is rational if and only if there is a periodic point in $A$.

- $\rho$ is irrational if and only if $\left.\varphi\right|_{A}$ is monotonically semiconjugate to the corresponding irrational rotation by $\rho$ on $\mathbb{T}^{1}$.

Barge and Gillette showed that the rotation number on a decomposable cofrontier is always unique, and it is rational if and only if there exists a periodic orbit in the cofrontier BG91. Theorem 1.1 complements these results to give a direct analogue to the Poincaré Classification Theorem for decomposable cofrontiers and, more generally, to circloids with decomposable boundary. Thus, dynamics with irrational rotation number on an invariant circloid with decomposable boundary are 'linearisable'.

As it further turns out, to a great extent this linearisation does not depend on the dynamics. More precisely, there exists a 'universal factor map' which maps the circloid to a topological circle and semiconjugates the dynamics of any homeomorphism preserving the circloid to that of a circle homeomorphism.

Theorem 1.2. Suppose that $A \subseteq \mathbb{A}$ is an essential circloid with decomposable boundary and there exists a self-homeomorphism of $\mathbb{A}$ leaving $A$ invariant without periodic points in $A$. Then there exists a continuous and onto map $\Pi: \mathbb{A} \rightarrow \mathbb{A}$ with the following properties.

(i) $\Pi$ is monotone and homotopic to the identity;

(ii) $\Pi$ sends $A$ to $\mathbb{T}=\mathbb{T}^{1} \times\{0\}$;

(iii) $\Pi$ is injective on $\mathbb{A} \backslash A$;

(iv) for any homeomorphism $\varphi: \mathbb{A} \rightarrow \mathbb{A}$ such that $\varphi(A)=A$ there exists a homeomorphism $\tilde{\varphi}: \mathbb{A} \rightarrow \mathbb{A}$ such that $\tilde{\varphi}(\mathbb{T})=\mathbb{T}$ and $\Pi \circ \varphi=\tilde{\varphi} \circ \Pi$.

(v) If $h: A \rightarrow \mathbb{T}^{1}$ is any monotone surjection, then there exists a monotone map $\widetilde{h}: \mathbb{T} \rightarrow \mathbb{T}^{1}$ such that $h=\tilde{h} \circ \Pi$. In particular, if $\varphi: \mathbb{A} \rightarrow \mathbb{A}$ is a homeomorphism leaving $A$ invariant and $h$ semiconjugates $\left.\varphi\right|_{A}$ to an irrational rotation $R_{\rho}$, then $\tilde{h}$ semiconjugates $\left.\tilde{\varphi}\right|_{\mathbb{T}}$ to $R_{\rho}$ (where $\tilde{\varphi}$ is as in the previous item).

We give a short self-contained proof in Section 5 It turns out, however, that the family of subcontinua of $A$ given by the fibres of $\Pi$ coincides with a decomposition of the circloid constructed already by Kuratowski, in a purely topological context Kur28. We discuss this in Section 7 .

It is well-known that the semiconjugacies in the Poincaré Classification Theorem are 
unique up to post-composition by a rotation. As an immediate consequence of Theorem 1.2 we obtain the same statement for decomposable circloids.

Corollary 1.3. The semiconjugacy in Theorem 1.1 is unique up to post-composition by a rotation.

It is worth mentioning that we do not know if an indecomposable cofrontier which is invariant by a homeomorphism may be semi-conjugate to an irrational rotation (however, if such an example exists the semiconjugacy cannot be monotone).

As should be expected, additional information on the topological structure of the circloid yields further information on the dynamics. We concentrate on the relation between the existence of biaccessible points and almost automorphic dynamics, whose study is a classical topic in abstract topological dynamics [Vee65, Ell69, Aus88. A homeomorphism $\varphi: X \rightarrow X$ is almost automorphic if it is semiconjugate to some almost periodic homeomorphism of a space $Y$ in a way that the set of points of $Y$ with a unique preimage under the semiconjugation is dense in $Y$. The following statement shows how sets of this type appear in surfaces. A point of an essential circloid $A \subset \mathbb{A}$ is called biaccessible if it is the unique intersection point of some arc $\sigma$ with $A$ such that $\sigma$ intersects both components of $\mathbb{A} \backslash A$. An essential cobasin boundary $B$ is the boundary of an essential circloid, $B=\partial A$, and if $A$ has a biaccessible point $x$ belonging to $B$, we also say that $x$ is a biaccessible point of $B$.

Theorem 1.4. If $B$ is an essential cobasin boundary in $\mathbb{A}$ invariant by a homeomorphism $\varphi: \mathbb{A} \rightarrow \mathbb{A}$ without periodic points and there is a biaccessible recurrent point in $B$, then $\left.\varphi\right|_{B}$ is almost automorphic.

Corollary 1.5. Let $\varphi: \mathbb{A} \rightarrow \mathbb{A}$ be a homeomorphism and $X$ is an essential $\varphi$-invariant continuum such that $\left.\varphi\right|_{X}$ is minimal. If $X$ has a biaccessible point, then $X$ is a decomposable cofrontier and $\left.\varphi\right|_{X}$ is almost automorphic.

We note that if a planar homeomorphism is both almost periodic and minimal on an invariant continuum $X$, then $X$ is a simple closed curve BGM93.

In MMO01, examples are constructed, using the Anosov-Katok method, of decomposable cofrontiers arising as the boundary of Siegel disks, which admit a monotone surjection onto $\mathbb{T}^{1}$ for which there is a subset of point inverses homeomorphic to the product of a cantor set and an interval (in particular, uncountably many fibers are nontrivial). All examples of minimal decomposable cofrontiers that we found in the literature are, to our knowledge, almost automorphic; see for instance Wal91, Her83. We close with a construction that is also based on the Anosov-Katok method AK70 and demonstrates that this is not necessarily the case: all fibres may be non-trivial.

Theorem 1.6. There exists a $C^{\infty}$ diffeomorphism $\varphi: \mathbb{A} \rightarrow \mathbb{A}$ leaving invariant a decomposable cofrontier $A$ such that

(i) the rotation number on $A$ is irrational;

(ii) the dynamics on $A$ are minimal;

(iii) all the fibres of points of $\mathbb{T}^{1}$ of the semiconjugacy given by Theorem 1.2 are nontrivial continua (i.e. not a single point).

In fact, the fibres of points of $\mathbb{T}^{1}$ have a diameter uniformly bounded below by a positive constant (see Claim 8.2 and, although we do not give a formal proof, it can be seen from the construction that all these fibres can be given a rich topological structure, reminiscent of the Knaster Buckethandle continuum.

We note that due to the nature of the construction, the rotation number of the example from Theorem 1.6 is Liouvillean. We do not know whether such an example exists with a Diophantine rotation number. Similar questions in the context of indecomposable cofrontiers have been raised by in [BGM93] (see also [Tur12]).

Acknowledgements. The authors are grateful to the anonymous referee for the suggestions that helped improve this paper. This work was initiated during the conference 'Surfaces in São Paulo', held in São Sebastião, 7-11 April 2014. TJ would like to thank the organisers and participants for creating this unique opportunity. This cooperation was supported by the Brasilian-European exchange program BREUDS (EU Marie Curie Action, IRSES Scheme). TJ acknowledges support of the German Research Council (Emmy 
Noether Grant Ja 1721/2-1). AK acknowledges support of CNPq-Brasil and FAPERJBrasil.

\section{Notation and preliminaries}

We denote by $\mathbb{A}=\mathbb{T}^{1} \times \mathbb{R}$ the open annulus, and $\pi: \mathbb{R}^{2} \rightarrow \mathbb{A}$ its universal covering map, where $T:(x, y) \mapsto(x+1, y)$ is a generator of the group of covering transformations.

A subset $A$ of the open annulus $\mathbb{A}=\mathbb{T}^{1} \times \mathbb{R}$ is called an essential annular continuum if it is compact and connected and its complement $\mathbb{A} \backslash A$ consists of exactly two connected components, both of which are unbounded. Note that in this situation one of the components is unbounded above and bounded below, whereas the other is bounded above and unbounded below, and both of them are homeomorphic to $\mathbb{A}$. Moreover, $A$ is the decreasing intersection of a sequence of closed annuli. We call $C \subseteq \mathcal{U}$ an essential circloid if it is a minimal element with respect to inclusion amongst essential annular continua. An essential circloid with empty interior is called an essential cofrontier. The boundary of an essential circloid is called an essential cobasin boundary. It is the intersection of the boundaries of the two complementary components of the circloid and a minimal element with respect to inclusion amongst essential continua. A subset $A$ of a surface $S$ is called annular continuum (circloid/cofrontier/cobasin boundary) if it has a neighbourhood $\mathcal{U}$ homeomorphic to $\mathbb{A}$ such that $A$ as a subset of $\mathcal{U}$ is an essential annular continuum (essential circloid/essential cofrontier/essential cobasin boundary) in the above sense. Note that thus an annular continuum in $\mathbb{A}$ may be non-essential, in which case it is contained in a closed topological disk. From now on, given any annular continuum, circloid, cobasin boundary or cofrontier, we always identify its annular neighbourhood $\mathcal{U}$ with $\mathbb{A}$ and assume implicitly that the objects are essential in $\mathbb{A}$.

A closed subset $\mathcal{A} \subseteq \mathbb{R}^{2}$ is called horizontal, if there exists $M>0$ with $\mathcal{A} \subseteq \mathbb{R} \times$ $[-M, M]$, and horizontally separating if $\mathbb{R} \times(-\infty,-M)$ and $\mathbb{R} \times(M, \infty)$ are contained in different connected components of $\mathbb{R}^{2} \backslash A$. It is called a horizontal strip if it separates the plane into exactly two connected components, one of them unbounded above and the other unbounded below. A horizontal strip is called minimal if it does not strictly contain a smaller horizontal strip. In this case, its boundary is called a horizontal coplane boundary and equals the intersection of the boundaries of the two complementary domains of the strip. A horizontal coplane boundary is minimal amongst horizontally separating sets. If $A$ is an essential continuum, we call the set $\mathcal{A}=\pi^{-1}(A)$ its lift. We state the next observation as a lemma, since it will be used repeatedly. Its proof is straightforward and left to the reader.

Lemma 2.1. The lift of an essential continuum $A$ is a minimal strip if and only if $A$ is a circloid, and the lift of an essential continuum $B$ is a coplane boundary if and only if $B$ is a cobasin boundary.

Let $\varphi: \mathbb{A} \rightarrow \mathbb{A}$ be a homeomorphism homotopic to the identity. Any such map lifts to a homeomorphism $\Phi: \mathbb{R}^{2} \rightarrow \mathbb{R}^{2}$ which commutes with the deck transformation $T$ : $(x, y) \mapsto(x+1, y)$. If $A$ is a compact invariant subset of $\varphi$, the rotation interval of $\Phi$ on $A$ is defined as

$$
\rho_{A}(\Phi)=\left\{\rho \in \mathbb{R} \mid \exists z_{i} \in \pi^{-1}(A), n_{i} \nearrow \infty: \lim _{i \rightarrow \infty} \pi_{1}\left(\Phi^{n_{i}}\left(z_{i}\right)-z_{i}\right) / n_{i}=\rho\right\} .
$$

When $\rho_{A}(\Phi)$ is reduced to a singleton $\{\rho\}$, we say $\Phi$ has a unique rotation number $\rho$ in $A$. In this case, $\lim _{n \rightarrow \infty} \pi_{1}\left(\Phi^{n}(z)-z\right) / n=\rho$ for all $z \in \pi^{-1}(A)$. When this happens we say that $\varphi$ has a well-defined rotation number $\rho(\varphi)=\rho+\mathbb{Z} \in \mathbb{T}^{1}$.

Given metric spaces $X, Y$, a continuous map $\varphi: X \rightarrow X$ is semiconjugate to $\psi: Y \rightarrow Y$ if there exists a continuous onto map $h: X \rightarrow Y$ such that $h \circ \varphi=\psi \circ h$. In this situation, we say $\psi$ is a factor of $\varphi$ and $h$ is a semiconjugacy or factor map. An important case is that of monotone semiconjugacies. A continuous map $h: X \rightarrow Y$ is called monotone if all fibres $h^{-1}(\{y\}), y \in Y$, are connected. A set $U \subseteq X$ is called saturated with respect to $h: X \rightarrow Y$, if $x \in U$ implies $h^{-1}(\{h(x)\}) \subseteq U$. If $h$ is continuous, then it maps saturated open (closed) sets to open (closed) sets. As a direct consequence, we have

Lemma 2.2. Preimages of connected sets under surjective monotone maps are connected. In particular, preimages of decomposable sets are decomposable. 
A cellular continuum in a surface $S$ is one of the form $K=\bigcap_{n \in \mathbb{N}} D_{n}$ where each $D_{n}$ is a closed topological disk and $D_{n+1} \subseteq \operatorname{int} D_{n}$. This is equivalent to saying that $K$ is a continuum and has a neighborhood homeomorphic to $\mathbb{R}^{2}$ in which $K$ is non-separating.

A partition $\mathcal{F}$ of a metric space $X$ into compact subsets is called an upper semicontinuous decomposition if for each open set $U \subseteq X$, the union of all elements of $\mathcal{F}$ contained in $U$ is also open. A Moore decomposition of a surface $S$ is an upper semicontinuous decomposition of $S$ into cellular continua. The following version of Moore's theorem is contained in Dav86, Theorem 25.1] (see also Theorem 13.4 in the same book). It says essentially that the quotient space of a Moore decomposition is the same surface $S$.

Theorem 2.3. Given any Moore decomposition $\mathcal{F}$ of a surface $S$, there exists a map $\Pi: S \rightarrow S$ which satisfies the following.

(i) $\Pi$ is continuous and surjective;

(ii) $\Pi$ is homotopic to the identity (and preserves orientation if $S$ is orientable);

(iii) For all $z \in S$, we have $\Pi^{-1}(z) \in \mathcal{F}$.

The map $\Pi$ is called the Moore projection associated to $\mathcal{F}$.

Finally, we state some basic results from plane topology. We say that a subset $K \subseteq X$ of a topological space separates two points if the two points belong to different connected components of $X \backslash K$.

Lemma 2.4 ([New92, Theorem 14.3]). If two points in the plane are separated by a closed set, then they are also separated by some connected component of that set.

Lemma 2.5 ([HY61, Theorem 2-28]). In any metric space, a continuum $K$ is homeomorphic to a circle if for any pair $x \neq y$ of points of $K$, the set $K \backslash\{x, y\}$ is disconnected.

Lemma 2.6 ([HY61, Theorem 2-16]). If $X$ is a continuum and $Y \subseteq X$ is closed, then the closure of every connected component of $X \backslash Y$ intersects $Y$.

\section{Minimal generators}

Throughout this section, $B \subseteq \mathbb{A}$ denotes a decomposable cobasin boundary and $\mathcal{B}=$ $\pi^{-1}(B)$ its lift. If $G \subseteq \mathcal{B}$ is a continuum such that $\mathcal{B}=\bigcup_{n \in \mathbb{Z}} T^{n}(G)$, we say that $G$ is a generator of $\mathcal{B}$. We say that $G$ is a minimal generator if it does not strictly contain a smaller generator. In the same way we may define generators and minimal generators for lifts of circloids. This concept has been used implicitly by Barge and Gillette in BG91; the terminology is taken from [JP]. As a consequence of Zorn's Lemma, any generator contains a minimal generator. The aim of this section is to provide a number of basic facts on minimal generators which will be crucial for the later constructions. The main objective is to derive the statements for circloids, but in order to do so first have to consider cobasin boundaries.

Lemma 3.1. A continuum $G \subseteq \mathcal{B}$ is a generator of $\mathcal{B}$ if and only if $G \cap T G \neq \varnothing$.

Proof. If $G \cap T G \neq \varnothing$, then $\bigcup_{k \in \mathbb{Z}} T^{k} G \subsetneq \mathcal{B}$ is horizontally separating and by Lemma 2.1 it has to be equal to $\mathcal{B}$, so $G$ is a generator. To prove the converse, we first note that if $G$ is a generator then $G \cap T^{k} G \neq \varnothing$ for some $k>0$, since otherwise $G$ would project injectively onto $B \subseteq \mathbb{A}$ contradicting the fact that $B$ is essential. If $k=1$ we are done; otherwise assume that $k$ is maximal with the property that $G \cap T^{k} G \neq \varnothing$, and note that $\bigcup_{i \in \mathbb{Z}} T^{i k} G$ is horizontally separating and so must be equal to $\mathcal{B}$; in particular it contains $T G$. But $T G \cap T^{i k} G=\varnothing$ for $i<0$ and $i>1$ due to the maximality of $k$. Thus $T G \subseteq G \cup T^{k} G$, and since $G$ is compact, $T G$ cannot be contained in $T^{k} G$, so $G \cap T G \neq \varnothing$ as claimed.

Lemma 3.2. Suppose that $L$ and $R$ are closed connected subsets of $\mathcal{B}$, with $L$ unbounded to the left and $R$ unbounded to the right. If $L \cap R=\varnothing$, then $\mathcal{B} \backslash(L \cup R)$ is connected, and if $L \cap R \neq \varnothing$ then $L \cup R=\mathcal{B}$.

Proof. If $L \cap R \neq \varnothing$, then $L \cup R$ is horizontally separating, so by Lemma 2.1 it must be equal to $\mathcal{B}$. Assume that $L \cap R=\varnothing$. Let $W^{-}$and $W^{+}$be the connected components of $\mathbb{R}^{2} \backslash \mathcal{B}$ which are unbounded below and above, respectively, so $\mathcal{B}=\partial W^{-}=\partial W^{+}$. Note that $L \cup R$ cannot be horizontally separating (since this would contradict Lemma 2.1); thus $W^{-}$and $W^{+}$are contained in the same connected component $U$ of $\mathbb{R}^{2} \backslash(L \cup R)$. 
Since $E:=\mathcal{B} \backslash(L \cup R) \subseteq \partial W^{-} \cap \partial W^{+}$, it follows that $E \subseteq U$. Note that $U$ is simply connected, since both $L$ and $R$ are connected and unbounded. Moreover, $E$ is a closed subset in the topology of $U$, and since $\mathcal{B}$ separates $W^{-}$from $W^{+}$in $\mathbb{R}^{2}$ it follows that $E=\mathcal{B} \cap U$ separates $W^{-}$from $W^{+}$in $U$. By Lemma 2.4 applied to $U \simeq \mathbb{R}^{2}$, some connected component $E_{0}$ of $E$ separates $W^{-}$from $W^{+}$in $U$. Since $E_{0}$ is closed in $U$, we have that $L \cup E_{0} \cup R$ is closed and horizontally separating, so by Lemma 2.1 it must be equal to $\mathcal{B}$. This implies that $E_{0}=E$, so $E$ is connected.

Lemma 3.3. There exists a minimal generator $G_{0}$ such that $G_{0} \cap T^{k} G_{0} \neq \varnothing$ if and only if $|k| \leqslant 1$. Moreover, $G_{0} \backslash T G_{0}$ and $G_{0} \backslash\left(T G_{0} \cup T^{-1} G_{0}\right)$ are connected and dense in $G_{0}$.

Proof. Since $B$ is decomposable, there exists a decomposition $B=X \cup Y$ into proper subcontinua. As $B$ is a cobasin boundary, both $X$ and $Y$ must be inessential in $\mathbb{A}$, which implies that there are open topological disks $D_{X} \supset X$ and $D_{Y} \supset Y$. Let $\widetilde{D}_{X}$ be a connected component of $\pi^{-1}\left(D_{X}\right)$, and $\mathcal{X}=\pi^{-1}(X) \cap \widetilde{D}_{X}$. Let $\widetilde{D}_{Y}$ be a connected component of $\pi^{-1}\left(D_{Y}\right)$, and $\mathcal{Y}=\pi^{-1}(X) \cap \widetilde{D}_{Y}$. The sets $\mathcal{X}$ and $\mathcal{Y}$ project injectively onto $X$ and $Y$, respectively, so they are continua and $T^{i} \mathcal{X} \cap \mathcal{X}=\varnothing$ for all $i \neq 0$, and similarly for $\mathcal{Y}$. Since $X \cap Y \neq \varnothing$, there exists $n \in \mathbb{Z}$ such that $T^{n} \mathcal{Y} \cap \mathcal{X} \neq \varnothing$. The set $\mathcal{X} \cup T^{n} \mathcal{Y}$ is a generator, so it contains some minimal generator $G_{0}$. Let $k$ be the largest integer such that $G_{0} \cap T^{k} G_{0} \neq \varnothing$, and suppose for a contradiction that $k>1$. Then $\bigcup_{i \in \mathbb{Z}, i \neq 1} T^{i} G_{0} \subseteq \bigcup_{i \in \mathbb{Z}, i \neq 1} T^{i} \mathcal{X} \cup T^{n+i} \mathcal{Y}$ is closed and horizontally separating, so in particular it contains $T \mathcal{X}$. Since $T \mathcal{X}$ is disjoint from $T^{i} \mathcal{X}$ for all $i \neq 1$, it follows that $T \mathcal{X} \subseteq \bigcup_{j \in \mathbb{Z}} T^{j} \mathcal{Y}$, which implies that $X=\pi(\mathcal{X}) \subset Y$, contradicting our choice of $X$ and $Y$.

By Lemma 3.2 we have that $E_{0}:=\mathcal{B} \backslash \bigcup_{k \neq 1} T^{k} G_{0}=G_{0} \backslash\left(T^{-1} G_{0} \cup T G_{0}\right) \neq \varnothing$ is connected. Note that the closure of any connected component of $G_{0} \backslash T G_{0}$ intersects $T G_{0}$ (see Lemma 2.6), so any connected component of $G_{0} \backslash T G_{0}$ must contain $E_{0}$ (otherwise it would be contained in $T^{-1} G_{0}$ which is disjoint from $T G_{0}$ ), so there is only one such component. Thus $G_{0} \backslash T G_{0}$ is connected. Since $\bigcup_{k \in \mathbb{Z}} T^{k}\left(G_{0} \backslash T G_{0}\right)=\mathcal{B}$, it follows that $\overline{G_{0} \backslash T G_{0}}$ is a generator and by minimality $G_{0}=\overline{G_{0} \backslash T G_{0}}$. This implies that $C=G_{0} \cap T G_{0}$ has empty interior in the restricted topology to $G_{0}$, and therefore $E_{0}=G_{0} \backslash\left(T^{-1} G_{0} \cup\right.$ $\left.T G_{0}\right)=G_{0} \backslash(C \cup T C)$ is also dense in $G_{0}$, completing the proof.

Lemma 3.4. If $G$ is any minimal generator of $\mathcal{B}$, then $T^{k} G \cap G \neq \varnothing$ if and only if $|k| \leqslant 1$. Moreover, $G \backslash T G$ and $G \backslash\left(T G \cup T^{-1} G\right)$ are connected and dense in $G$.

Proof. Suppose that $T^{k} G \cap G \neq \varnothing$ for some $k>1$, so there exists $z \in G$ such that $T^{k} z \in G$. Let $G_{0}$ be as in Lemma 3.3 Replacing $G$ by $T^{i} G$ for a suitable $i$, we may assume that $z \in T^{-1} G_{0}$. This means that $G$ intersects $T^{-1} G_{0}$ and $T^{k-1} G_{0}$, where $k-1 \geqslant 1$. Thus the set $G \cup \bigcup_{k \neq 0} T^{i} G_{0}$ is closed, connected and horizontally separating, and by Lemma 2.1 it should be equal to $\mathcal{B}$. Thus $G_{0} \backslash\left(T^{-1} G_{0} \cup T G_{0}\right)=G_{0} \backslash \bigcup_{k \neq 0} T^{i} G_{0} \subseteq G$, implying that $G_{0} \subseteq G$. By minimality $G_{0}=G$, contradicting the fact that $G \cap T^{k} G \neq \varnothing$ with $k>1$.

Knowing that $G \cap T^{k} G \neq \varnothing \Longleftrightarrow|k| \leqslant 1$, the remaining claims are proved exactly as in the last paragraph of the proof of Lemma 3.3 .

Given a minimal generator $G$ of $\mathcal{B}$, let $\mathcal{L}_{n}(G)=\bigcup_{k \leqslant n} T^{k}(G)$ and $\mathcal{R}_{n}(G)=\bigcup_{k \geqslant n} T^{k}(G)$. With these notions, we have

Lemma 3.5. If $G$ and $G^{\prime}$ are two different minimal generators of $\mathcal{B}$, then either $G^{\prime} \subseteq$ $\mathcal{B} \backslash \mathcal{L}_{-1}(G) \subseteq \mathcal{R}_{0}(G)$ or $G^{\prime} \subseteq \mathcal{B} \backslash \mathcal{R}_{1}(G) \subseteq \mathcal{L}_{0}(G)$.

Proof. Suppose $G^{\prime}$ intersects both $\mathcal{L}_{-1}(G)$ and $\mathcal{R}_{1}(G)$. Then $\mathcal{L}_{-1}(G) \cup G^{\prime} \cup \mathcal{R}_{1}(G)=\mathcal{B}$ by Lemma 2.1, so the union contains $G$. Since $G \backslash\left(T G \cup T^{-1} G\right)$ is disjoint from $\mathcal{L}_{-1}(G) \cup$ $\mathcal{R}_{1}(G)$, we have that $G \backslash\left(T G \cup T^{-1} G\right) \subseteq G^{\prime}$, which implies by Lemma 3.4 that $G \subseteq G^{\prime}$, so by the minimality $G=G^{\prime}$.

Corollary 3.6. If $G$ and $G^{\prime}$ are minimal generators of $\mathcal{B}$, then $G^{\prime}$ is contained in two adjacent copies of $G$ and vice versa.

A cut (of $\mathcal{B}$ ) is a set of the form $G \cap T^{-1} G$ where $G$ is a minimal generator of $\mathcal{B}$. We denote by $\mathcal{C}$ the family of all cuts. Note that by Lemma 3.5. cuts are pairwise disjoint.

Given a cut $C=G \cap T^{-1}(G)$, we let $\widehat{\mathcal{R}}(C)=\mathcal{R}_{0}(G)$ and $\widehat{\mathcal{L}}(C)=\mathcal{L}_{-1}(G)$, so that $C=\widehat{\mathcal{R}}(C) \cap \widehat{\mathcal{L}}(C)$. Further, we let $\mathcal{L}(C)=\hat{\mathcal{L}}(C) \backslash C$ and $\mathcal{R}(C)=\hat{\mathcal{R}}(C) \backslash C$. We write $C \prec C^{\prime}$ if $C \subseteq \mathcal{L}\left(C^{\prime}\right)$, or equivalently if $C^{\prime} \subseteq \mathcal{R}(C)$. By Lemma 3.5 and its corollary, < 
defines a total order in $\mathcal{C}$. We extend this notation to compare arbitrary subsets $S \subseteq \mathcal{B}$ with cuts by writing $S<C$ if $S \subseteq \mathcal{L}(C)$ and $S \leqslant C$ if $S \subseteq \hat{\mathcal{L}}(C)$. If $z \in \mathcal{B}$ and $\{z\}<C$, we simply write $z<C$. For two cuts $C<C^{\prime}$, we let $\left(C, C^{\prime}\right)_{\mathcal{B}}=\mathcal{R}(C) \cap \mathcal{L}\left(C^{\prime}\right)=\{z \in \mathcal{B} \mid$ $\left.C<z<C^{\prime}\right\}$ and $\left[C, C^{\prime}\right]_{\mathcal{B}}=\widehat{\mathcal{R}}(C) \cap \widehat{\mathcal{L}}\left(C^{\prime}\right)=\left(C, C^{\prime}\right)_{\mathcal{B}} \cup C \cup C^{\prime}$.

We note that cuts need not be connected. However, we have:

Lemma 3.7. Given two cuts $C \prec C^{\prime}$, the set $\left(C, C^{\prime}\right)_{\mathcal{B}}$ is connected and its closure is $\left[C, C^{\prime}\right]_{\mathcal{B}}$.

Proof. We have $\left(C, C^{\prime}\right)_{\mathcal{B}}=\mathcal{B} \backslash\left(\mathcal{L}_{0}(G) \cup \mathcal{R}_{0}\left(G^{\prime}\right)\right) \neq \varnothing$ for some minimal generators $G, G^{\prime}$. The connectedness follows from Lemma 3.2 and the fact that its closure is $\left[C, C^{\prime}\right]_{\mathcal{B}}$ follows easily from Lemma 3.4

Let $A$ be the essential circloid such that $\partial A=B$ (i.e. $A$ is the union of $B$ with all bounded connected components of $\mathbb{A} \backslash B)$, and let $\mathcal{A}=\pi^{-1}(A)$ be its lift. A generator of $\mathcal{A}$ is a continuum $G \subseteq \mathcal{A}$ which satisfies $\bigcup_{n \in \mathbb{Z}} T^{n} G=\mathcal{A}$. In order to go over from a the decomposable cobasin boundary $B$ to the corresponding circloid $A$, the following statements will be crucial.

Lemma 3.8. All connected components of $\mathcal{A} \backslash \mathcal{B}$ are topological disks with diameter bounded by a uniform constant $M$.

Proof. Let $G$ be a generator of $\mathcal{B}$, and suppose $\pi_{1}(G)=[a, b]$. Let $N \in \mathbb{N}$ be such that $a+N>b$. Since $\pi_{2}(G)=\pi_{2}(\mathcal{A})$, the latter set has diameter bounded by some constant c. let $M=2 N+c+1$. If $U$ is a connected component of $\mathcal{A} \backslash \mathcal{B}$ with $\operatorname{diam}(U)>M$, then $\operatorname{diam}\left(\pi_{1}(U)\right)>2 N+1$ and we may assume $[a-N, b+N] \subseteq \pi_{1}(U)$ replacing $U$ by $T^{i} U$ for an appropriate $i \in \mathbb{Z}$. Thus there is a simple arc $\gamma:[0,1] \rightarrow U$ such that $\pi_{1}(\gamma(0))=a-N, \pi_{1}(\gamma(1))=b+N$, and $a-N<\gamma(t)<b+N$ for $0<t<1$. If $K=\gamma([0,1])$ and $S:=[a-N, b+N] \times \mathbb{R}$, we have that $S \backslash K$ has exactly two connected components $S_{+}$and $S_{-}$, the former unbounded above and the later unbounded below. The set $G^{\prime}=\bigcup_{k=-N}^{N} T^{k} G$ being connected, disjoint from $K$ and contained in $S$, must lie entirely in $S^{-}$or $S^{+}$. Suppose without loss of generality that $G^{\prime} \subseteq S^{-}$. Note that $K \subseteq U$ and $U$ is is bounded above. If $y$ is the smallest real such that $\{a\} \times[y, \infty) \subseteq \mathbb{R}^{2} \backslash \mathcal{B}$, then $\{a\} \times[y, \infty)$ is disjoint from $K$ and thus contained in $S^{+}$, and since $z:=(a, y) \in \partial \mathcal{B}$ there must exist $n$ such that $z \in T^{n} G$. Since $T^{k} G \subseteq S^{-}$when $|k| \leqslant N$, it follows that $n>N$, but this is not possible since $\pi_{1}\left(T^{n} G\right) \subseteq[a+N, \infty) \subseteq(b, \infty)$.

Given a continuum $S \subseteq \mathbb{R}^{2}$, the complement $\mathbb{R}^{2} \backslash S$ consists of one unbounded component and a union of topological disks. We denote the unbounded component by $U_{\infty}(S)$ and the family of disks by $\mathcal{U}_{S}$ and let $S^{\text {fill }}=\mathbb{R}^{2} \backslash U_{\infty}(S)=S \cup \bigcup_{U \in \mathcal{U}_{S}} U$. Note that $S^{\text {fill }}$ is a nonseparating continuum.

Lemma 3.9. Suppose $U$ is a connected component of $\mathcal{A} \backslash \mathcal{B}$ and $C_{0}^{-}<C_{0}^{+}$are cuts such that $\left(C_{0}^{-}, C_{0}^{+}\right)_{\mathcal{B}} \cap \partial U \neq \varnothing$. If $C^{-}, C^{+}$are cuts with $C^{-}<C_{0}^{-}<C_{0}^{+}<C^{+}$, then $\partial U \subseteq\left[C^{-}, C^{+}\right]_{\mathcal{B}}$.

Proof. Suppose for a contradiction that $\partial U \nsubseteq\left[C^{-}, C^{+}\right]_{\mathcal{B}}$. Assume without loss of generality that $\partial U \cap \mathcal{L}\left(C^{-}\right) \neq \varnothing$. Since $U$ is bounded, there is $n>0$ such that $\partial U \subseteq\left[T^{-n} C^{-}, T^{n} C^{-}\right]_{\mathcal{B}}$, and we may assume $T^{n} C^{-}<C_{0}^{-}$and $C_{0}^{+}<T^{n} C^{-}$. The sets $L=\left[T^{-n} C^{-}, C_{0}^{-}\right]_{\mathcal{B}}$ and $R=\left[C^{-}, T^{n} C^{-}\right]_{\mathcal{B}}$ are connected, and $L \cap R=\left[C_{0}^{-}, C^{-}\right]_{\mathcal{B}}$ is also connected (by Lemma 3.7). Since $\partial U$ is not contained in either set $L$ or $R$, we have that $U \subseteq U_{\infty}(L) \cap U_{\infty}(R)$. But then by Lemma 2.4 we have that $U \subseteq U_{\infty}(R \cup L)$, contradicting the fact that $U$ is bounded and $\partial U \subseteq L \cup R$.

\section{Dynamical linearisation: Proof of Theorem 1.1}

Throughout this section, we assume that $\varphi: \mathbb{A} \rightarrow \mathbb{A}$ is a homeomorphism homotopic to the identity and $A$ is a $\varphi$-invariant circloid with decomposable boundary. We let $B=\partial B$ and denote the lifts of $A, \varphi$ and $B$ by $\mathcal{A}, \Phi$ and $\mathcal{B}$, respectively. The next result generalizes [BG91, Theorem 2.7] to circloids.

Lemma 4.1. The rotation number $\rho(\Phi, \mathcal{A})=\lim _{n \rightarrow \infty} \pi_{1}\left(\Phi^{n}(z)-z\right) / n$ exists and is independent of $z \in \mathcal{A}$. 
Proof. The fact that $\rho=\lim _{n \rightarrow \infty} \pi_{1}\left(\Phi^{n}\left(z_{0}\right)-z_{0}\right) / n$ exists for some $z_{0} \in \mathcal{B}$ follows from the Birkhoff Ergodic Theorem and the existence of an invariant measure for $\left.\varphi\right|_{B}$, since $\pi_{1}\left(\Phi^{n}\left(z_{0}\right)-z_{0}\right) / n$ is a Birkhoff average for the function $B \ni x \mapsto \pi_{1}\left(\Phi\left(x^{\prime}\right)-x^{\prime}\right) \in \mathbb{R}$, where $x^{\prime} \in \pi^{-1}(x)$ is arbitrary.

Fix a minimal generator $G$ of $\mathcal{B}$ containing $z_{0}$. Since $\Phi^{n}(G)$ is also a minimal generator, by Corollary 3.6 there exists $k$ such that $\Phi^{n}(G) \subset T^{k}(G \cup T G)$, so $\operatorname{diam}\left(\Phi^{n}(G)\right) \leqslant$ $2 \operatorname{diam}(G):=M$ for all $n \in \mathbb{N}$. This implies $\lim _{n \rightarrow \infty} \pi_{1}\left(\Phi^{n}(z)-z\right) / n=\rho$ for all $z \in G$, and since $G$ is a generator the same holds for all $z \in \mathcal{B}$. Finally, we deduce from Lemma 3.8 that the same property holds for $z \in \mathcal{A}$.

Lemma 4.2. $\rho(\Phi, \mathcal{A})=p / q \in \mathbb{Q}$ if and only if there exists $z \in \mathcal{A}$ such that $\Phi^{q}(z)=T^{p} z$.

Proof. The if-part is trivial. For the other implication, note that it is easy to verify that $\rho\left(T^{-p} \Phi^{q}, \mathcal{A}\right)=0$ if and only if $\rho(\Phi, \mathcal{A})=p / q$, so it suffices to assume that $\rho(\Phi, \mathcal{A})=0$ and show that there is a fixed point in $\mathcal{A}$. Fix a minimal generator $G$ of $\mathcal{B}$. We claim that $\Phi^{n}(G) \cap G \neq \varnothing$ for all $n$. Indeed, fix a positive integer $n$. Corollary 3.6 implies that $\Phi^{n}(G) \subset T^{k} G \cup T^{k+1} G$ for some $k$. If $k>0$, then $\Phi^{n}(G) \subset \mathcal{R}_{1}(G)$ which then implies $\Phi^{n k}(G) \subset \mathcal{R}_{k}(G)$ and this implies that $\pi_{1}\left(\Phi^{n k}(z)-z\right) / n k \geqslant 1 / n$ for all $k \in \mathbb{N}$ and $z \in G$, contradicting our assumption. If $k<-1$, we get a similar contradiction. Thus $k \in\{0,-1\}$, and since $G$ and $\Phi^{n}(G)$ are minimal generators, $\Phi^{n}(G)$ must intersect both $T^{k} G$ and $T^{k+1} G$, so $\Phi^{n}(G) \cap G \neq \varnothing$ as claimed. Thus $K=\overline{\bigcup_{n \in \mathbb{Z}} \Phi^{n}(G)} \subset \mathcal{B}$ is connected and bounded (again due to Corollary 3.6), and $\Phi(K)=K$. Moreover, $K^{\text {fill }} \subset \mathcal{A}$ and $\Phi\left(K^{\text {fill }}\right)=K^{\text {fill }}$, so $K^{\text {fill }}$ is a non-separating invariant continuum in $\mathbb{R}^{2}$ and the generalization of the Cartwright-Littlewood theorem due to Bell [Bel78] implies that $K^{\text {fill }}$ contains a fixed point of $\Phi$.

The previous lemma implies the first item from Theorem 1.1 It also follows that if $\left.\varphi\right|_{A}$ is monotonically semiconjugate to an irrational rotation then $\rho(\Phi, \mathcal{A})$ is irrational. Thus to complete the proof of the theorem it remains to prove that if $\rho=\rho(\Phi, \mathcal{A})$ is irrational, then $\left.\varphi\right|_{A}$ is monotonically conjugate to the corresponding irrational rotation.

For the remainder of this section we assume that $\rho$ is irrational, and we fix a minimal generator $G_{0}$ of $\mathcal{B}$. Given $x=n+k \rho$ in $Q(\rho)=\{l+\rho m \mid l, m \in \mathbb{Z}\}$, we let $G_{x}=\Phi^{k} \circ T^{n}\left(G_{0}\right)$ and denote by $C_{x}=G_{x} \cap T^{-1}\left(G_{x}\right)$ the cut corresponding to $G_{x}$. Recall that there is a linear ordering $\prec$ on the set $\mathcal{C}$ of cuts as defined in the previous section.

Lemma 4.3. The mapping $(Q(\rho),<) \rightarrow(\mathcal{C}, \prec), x \mapsto C_{x}$ is strictly monotonically increasing. In particular, $C_{x} \cap C_{y}=\varnothing$ if $x \neq y$. Moreover, the set $\left[C_{x}, C_{y}\right]_{\mathcal{B}}$ is connected for all $x<y$, decreasing in $x$ and increasing in $y$.

Proof. Suppose that $x=n+k \rho<x^{\prime}=n^{\prime}+k^{\prime} \rho$, but $C_{x^{\prime}} \preccurlyeq C_{x}$. Then $\Phi^{k^{\prime}-k} \circ$ $T^{n^{\prime}-n}\left(\widehat{\mathcal{L}}\left(C_{0}\right)\right) \subseteq \widehat{\mathcal{L}}\left(C_{0}\right)$. As a consequence, all orbits in $\widehat{\mathcal{L}}\left(C_{0}\right)$ under the lift $\Psi=\Phi^{k^{\prime}-k} \circ$ $T^{n^{\prime}-n}$ of $\varphi^{k^{\prime}-k}$ are bounded to the right, contradicting the fact that $\rho(\Psi)=x^{\prime}-x>0$. This shows the strict monotonicity of $x \mapsto C_{x}$ and the disjointness. Connectedness of $\left[C_{x}, C_{y}\right]_{\mathcal{B}}$ is given by Lemma 3.7

As in the previous section, given $z \in \mathcal{B}$ and a cut $C \in \mathcal{C}$, we write $z<C$ iff $z \in \mathcal{L}(C)$ and $C \prec z$ iff $z \in \mathcal{R}(C)$. In order to extend this notion to all $z \in \mathcal{A}$, note that $\mathcal{A} \backslash \mathcal{B}$ is a union of bounded open topological disks whose boundary is contained in $\mathcal{B}$. Given $z \in \mathcal{A} \backslash \mathcal{B}$, we denote the respective disk containing $z$ by $U_{z}$ and write $z<C$ iff $\partial U_{z} \subseteq \mathcal{L}(C)$ and $C \prec z$ iff $\partial U_{z} \subseteq \mathcal{R}(C)$. Equivalently, $z \prec C$ iff $z \in \mathcal{L}(C)^{\text {fill }}$ and $C \prec z$ iff $z \in \mathcal{R}(C)^{\text {fill }}$. Given a subset $S \subseteq \mathcal{A}$, we write $C \prec S$ iff $S \subseteq \mathcal{L}(C)^{\text {fill }}$ and $S<C$ iff $S \subseteq \mathcal{R}(C)^{\text {fill. }}$. Then, we define $H: \mathcal{A} \rightarrow \mathbb{R}$ by

$$
H(z)=\sup \left\{x \in Q(\rho) \mid C_{x} \prec z\right\} .
$$

Lemma 4.4. The map $H$ is continuous and projects to a monotone semiconjugacy $h$ : $A \rightarrow \mathbb{T}^{1}$ from $\left.\varphi\right|_{A}$ to the irrational rotation by $\rho$.

Proof. We first show the continuity of the restriction of $H$ to $\mathcal{B}$. By definition, we have that

$$
H_{\mid \mathcal{B}}^{-1}((x, y))=\bigcup_{\substack{x^{\prime}, y^{\prime} \in Q(\rho) \\
x<x^{\prime}<y^{\prime}<y}}\left(C_{x^{\prime}}, C_{y^{\prime}}\right)_{\mathcal{B}}=\bigcup_{\begin{array}{c}
x^{\prime}, y^{\prime} \in Q(\rho) \\
x<x^{\prime}<y^{\prime}<y
\end{array}}\left[C_{x^{\prime}}, C_{y^{\prime}}\right]_{\mathcal{B}}
$$


Since the sets $\left(C_{x^{\prime}}, C_{y^{\prime}}\right)_{\mathcal{B}}$ are relatively open in $\mathcal{B}$, this shows that preimages of open sets are open, so that $H_{\mid \mathcal{B}}$ is continuous.

In order to see that $H$ is continuous on all of $\mathcal{A}$, fix $x<y$ and $z \in H^{-1}((x, y))$. It suffices show to that $H^{-1}((x, y))$ contains a neighbourhood of $z$. If $z \in \mathcal{A} \backslash \mathcal{B}$, then by definition the whole open disk $U_{z}$ is contained in $H^{-1}((x, y))$. (Note that if $C_{x} \prec z$, then $C_{x} \prec z^{\prime}$ for all $z^{\prime} \in U_{z}$.) Thus, suppose that $z \in \mathcal{B}$. Since $H_{\mid \mathcal{B}}$ is continuous, there exists $\varepsilon>0$ such that $B_{\varepsilon}(z) \cap \mathcal{B} \subseteq H^{-1}((x, y))$. If $z^{\prime} \in B_{\varepsilon}(z) \cap(\mathcal{A} \backslash \mathcal{B})$, then $B_{\varepsilon}(z)$ intersects both $\mathcal{B}$ and $U_{z^{\prime}}$. Consequently, $B_{\varepsilon}(z)$ intersects $\partial U_{z^{\prime}}$ and we have $\varnothing \neq \partial U_{z^{\prime}} \cap B_{\varepsilon}(z) \subseteq$ $H^{-1}((x, y))$. However, this implies that for some $x^{\prime}, y^{\prime}$ with $x<x^{\prime}<y^{\prime}<y$ we have $\partial U_{z^{\prime}} \cap\left(C_{x^{\prime}}, C_{y^{\prime}}\right)_{\mathcal{B}} \neq \varnothing$. Therefore Lemma 3.9 yields that $\partial U_{z^{\prime}} \subseteq\left[C_{\tilde{x}}, C_{\tilde{y}}\right]_{\mathcal{B}}$ for any $\tilde{x}, \tilde{y}$ with $x<\tilde{x}<x^{\prime}<y^{\prime}<\tilde{y}<x$ and thus $U_{z^{\prime}} \subseteq H^{-1}((x, y))$. Altogether, we obtain $B_{\varepsilon}(z) \cap \mathcal{A} \subseteq H^{-1}((x, y))$, which proves the continuity of $H$ on $\mathcal{A}$.

In order to show the further statements, note that since by definition $\Phi\left(G_{x}\right)=G_{x+\rho}$ and $T\left(G_{x}\right)=G_{x+1}$, the same relations hold for $C_{x}$ and $\mathcal{L}\left(C_{x}\right)$. Using these facts, it is easy to check that $H$ is a semiconjugacy from $\Phi_{\mid \mathcal{A}}$ to the translation $x \mapsto x+\rho$ on $\mathbb{R}$ and that $H$ commutes with the deck translation $T$, such that $H$ projects to a semiconjugacy $h$ from $\varphi_{\mid A}$ to the rotation $R_{\rho}$.

It remains to prove the monotonicity of $h$, which will follow immediately from that of $H$. We have that

$$
H_{\mid B}^{-1}(x)=\bigcap_{\substack{x^{\prime}, y^{\prime} \in Q(\rho) \\ x^{\prime}<x<y^{\prime}}}\left[C_{x^{\prime}}, C_{y^{\prime}}\right] .
$$

This can be seen as a nested intersection of continua and is therefore a continuum itself. The full fibre $H^{-1}(x)$ is obtained by adding the union $\bigcup_{z \in H^{-1}(x) \backslash \mathcal{B}} U_{z}$ of topological open disks to $H_{\mid B}^{-1}(x)$. However, if $z \in H^{-1}(x)$, then $\partial U_{z}$ cannot intersect $\mathcal{L}\left(C_{x^{\prime}}\right)$ for any $x^{\prime}<x$, since otherwise Lemma 3.9 would imply that $\partial U_{z} \subseteq \widehat{\mathcal{L}}\left(C_{\tilde{x}}\right)$ for some $\tilde{x} \in\left(x^{\prime}, x\right)$ and thus $H(z) \leqslant \tilde{x}$. Similarly, $\partial U_{z}$ is disjoint from $\mathcal{R}\left(C_{y^{\prime}}\right)$ for all $y^{\prime}>x$, and therefore $\partial U_{z} \subseteq H^{-1}(x)$. Hence, we have that $H^{-1}(x)=H_{\mathcal{B}}^{-1}(x)^{\text {fill }}$ is the 'fill-in' of a continuum, and hence a continuum itself.

This concludes the proof of Theorem 1.1

\section{Topological linearisation: a universal factor map}

The main goal of this section is to prove Theorem 1.2 We refer the reader to Section 7 for a discussion on the relationship between the content presented here and the work of Kuratowski.

As before, we suppose $A \subseteq \mathbb{A}$ is a circloid with decomposable boundary, and $\mathcal{A}=$ $\pi^{-1}(A)$. We let $B=\partial A$ and $\mathcal{B}=\pi^{-1}(B)$. Our aim is to define a Moore decomposition of $\mathbb{A}$ such that the corresponding projection maps $A$ to a topological circle. As before, we start by decomposing $B$, and we use the family $\mathcal{C}$ of cuts of $\mathcal{B}$ as the main tool.

However, cuts need not be connected, and moreover is easy to give examples where $\bigcup_{C \in \mathcal{C}} C$ does not cover all of $\mathcal{B}$. In order to obtain a decomposition starting from $\mathcal{C}$, we define a strong partial order relation $\ll$ on $\mathcal{C}$ by writing $C \ll C^{\prime}$ if and only if there exist uncountably many cuts $\tilde{C} \in \mathcal{C}$ such that $C \prec \tilde{C} \prec C^{\prime}$. Similar to before, we extend this definition to arbitrary subsets $S, S^{\prime} \subseteq \mathcal{A}$ by writing $S \ll S^{\prime}$ whenever there exist uncountably many cuts $\tilde{C}$ with $S<\tilde{C} \prec S^{\prime} \bigsqcup^{2}$ In case of one-point sets $S=\{z\}$, we write $z \ll S^{\prime}$ instead of $\{z\} \ll S^{\prime}$. Then, given any $z \in \mathcal{B}$, we define

$$
F(z)=\bigcap_{\substack{C^{-}, C+\in \mathcal{C} \\ C^{-} \ll z \ll C^{+}}}\left[C^{-}, C^{+}\right]_{\mathcal{B}} .
$$

We let $\mathcal{F}_{\mathcal{B}}=\{F(z) \mid z \in \mathcal{B}\}$ and call the elements $F \in \mathcal{F}_{\mathcal{B}}$ fibres of $\mathcal{B}$. Further, we let $\mathcal{F}_{B}=\left\{\pi(F) \mid F \in \mathcal{F}_{\mathcal{B}}\right\}$. We note that the intersection in 5.1] can be viewed as a nested intersection of continua: by compactness, for every $n \in \mathbb{N}$ there exist $C_{n}^{-} \ll z \ll C_{n}^{+}$such

\footnotetext{
${ }^{2}$ We note that the requirement of uncountably many intermediate cuts is crucial for the whole construction in this section. We do not elaborate further on this, but just mention that a examples demonstrating why requiring uncountably many intermediate cuts is necessary can be produced by gluing finitely or countably many pseudoarcs together. Otherwise, a statement analogous to Lemma 5.1 does not hold.
} 
that $F(z) \subseteq\left[C_{n}^{-}, C_{n}^{+}\right] \subseteq B_{1 / n}(F(z))$. Without loss of generality we may assume that $C_{n}^{-} \ll C_{n+1}^{-}$and $C_{n+1}^{+} \ll C_{n}^{+}$for all $n \in \mathbb{N}$, and we have $F(z)=\bigcap_{n \in \mathbb{N}}\left[C_{n}^{-}, C_{n}^{+}\right]$. By Lemma 3.7 this is a nested intersection of continua, hence a continuum.

Lemma 5.1. If $S, S^{\prime} \subseteq \mathcal{B}$ and $S \ll S^{\prime}$, then there exists $C \in \mathcal{C}$ such that $S \ll C \ll S^{\prime}$.

Proof. We first show the following slightly weaker

Claim 5.2. If $V, V^{\prime} \subseteq \mathcal{A}$ and $V \ll V^{\prime}$, then there exist $C, C^{\prime} \in \mathcal{C}$ such that $V \ll C \prec V^{\prime}$ and $V \prec C^{\prime} \ll V^{\prime}$.

We will prove the existence of $C$; that of $C^{\prime}$ then follows by symmetry. Suppose for a contradiction that for all $C \in \mathcal{A}$ with $V \prec C \prec V^{\prime}$ we have $V \nVdash C$. Let $R=\bigcap_{C \in \mathcal{C}, C<V^{\prime}} \hat{\mathcal{R}}(C)$ and choose an increasing sequence of cuts $C_{n}<V^{\prime}$ with $R=$ $\bigcap_{n \in \mathbb{N}} \widehat{\mathcal{R}}\left(C_{n}\right)$. Note that, for example, it suffices to choose $C_{n}$ such that $\operatorname{dist}_{\mathcal{H}}\left(\widehat{\mathcal{R}}\left(C_{n}\right), R\right)<$ $1 / n$. Then, since $V \nVdash C_{n}$, there exist at most countably many cuts between $V$ and $C_{n}$. However, every cut between $V$ and $V^{\prime}$ is either equal to $C_{n}$ for some $n \in \mathbb{N}$, lies between $V$ and $C_{1}$ or lies between $C_{n}$ and $C_{n+1}$ for some $n \in \mathbb{N}$. Altogether, we obtain that there are at most countably many cuts between $V$ and $V^{\prime}$, contradicting $V \ll V^{\prime}$. This proves the claim.

Now, suppose for a contradiction that for every cut $C$ between $S$ and $S^{\prime}$ we either have $S \nVdash C$ or $C \nVdash S^{\prime}$. Note that both properties cannot hold simultaneously since $S \ll S^{\prime}$. Let $L^{\prime}=\bigcap_{C \in \mathcal{C}, S \ll C} \hat{\mathcal{L}}(C)$ and $R^{\prime}=\bigcap_{C^{\prime} \in \mathcal{C}, C^{\prime} \ll S^{\prime}} \hat{\mathcal{R}}\left(C^{\prime}\right)$. Then the intersection $E=L^{\prime} \cap R^{\prime}$ is non-empty, since for every pair of cuts $C, C^{\prime}$ with $C^{\prime} \ll S^{\prime}$ and $S \ll C$ we have $C^{\prime}<C$ due to our contradiction assumption. Moreover, $G=T\left(L^{\prime}\right) \cap R^{\prime}$ is a continuum, since it can again be represented as a nested intersection of intervals in $\mathcal{B}$. Since $G \cap T^{-1}(G)=E \neq \varnothing$, it follows from Lemma 3.1 that $G$ is a generator. Thus, it contains a minimal generator, and consequently the set $E$ contains some cut $\widetilde{C}$. If $S \ll \widetilde{C}$, then Claim 5.2 implies that there exists $C_{1} \in \mathcal{C}$ such that $S \ll C_{1} \prec \widetilde{C}$, and since $\widetilde{C} \subseteq L^{\prime}$ it follows that $\widetilde{C} \subseteq \widehat{\mathcal{L}}\left(C_{1}\right)$, contradicting the fact that $C_{1} \prec \widetilde{C}$. Thus $S \nVdash \widetilde{C}$, and by a similar argument $\widetilde{C} \ll S^{\prime}$. However, this contradicts the fact that $S \ll S^{\prime}$.

Lemma 5.3. Distinct fibres $F, F^{\prime} \in \mathcal{F}_{\mathcal{B}}$ are disjoint and either $F \ll F^{\prime}$ or $F^{\prime} \ll F$.

Proof. Let $F=F(z)$ and $F^{\prime}=F\left(z^{\prime}\right)$. If $F \neq F^{\prime}$, then there exists $C \in \mathcal{C}$ such that either $z \ll C$ and $z^{\prime} \nVdash C$, or $C \ll z$ and $C \nVdash z^{\prime}$. Assume the former case (the other case is analogous). According to Lemma 5.1 there exist $C^{-}, C^{+}$with $z \ll C^{-} \ll C^{+} \ll C$. However, this implies that $z \ll C^{-} \ll C^{+} \ll z^{\prime}$, hence $F(z) \subseteq \widehat{\mathcal{L}}\left(C^{-}\right)$and $F\left(z^{\prime}\right) \subseteq \widehat{\mathcal{R}}\left(C^{+}\right)$ are disjoint and $F(z) \ll F\left(z^{\prime}\right)$.

We now turn to the decomposition of $\mathcal{A}$. As before, given $z \in \mathcal{A} \backslash \mathcal{B}$, we denote by $U_{z}$ the connected component of $\mathcal{A} \backslash \mathcal{B}$ containing $z$.

Lemma 5.4. Suppose $F \in F_{\mathcal{B}}$ intersects $\partial U_{z}$ for some $z \in \mathcal{A} \backslash \mathcal{B}$. Then $\partial U_{z} \subseteq F$.

Proof. Suppose $F=F\left(z^{\prime}\right)$ intersects $\partial U_{z}$. Then given any cuts $C^{-} \ll z \ll C^{+}$, Lemma 5.1 yields cuts $C_{0}^{-}, C_{0}^{+}$with $C^{-} \ll C_{0}^{-} \ll F \ll C_{0}^{+} \ll C^{+}$and $\partial U_{z} \cap\left(C_{0}^{-}, C_{0}^{+}\right)_{\mathcal{B}} \neq \varnothing$. By Lemma 3.9 this implies that $\partial U_{z} \subseteq\left[C^{-}, C^{+}\right]_{\mathcal{B}}$. Since this is true for all pairs of cuts satisfying $C^{-} \ll z \ll C^{+}$, we obtain $\partial U_{z} \subseteq F$.

Bounded connected components of the complement of a fibre $F \in \mathcal{F}_{\mathcal{B}}$ are also bounded connected components of $\mathcal{A} \backslash \mathcal{B}$. Therefore, the preceding lemma implies that $F^{\text {fill }}=$ $F \cup \bigcup_{\partial U_{z} \cap F \neq \varnothing} U_{z}$. This allows to define a decomposition of $\mathcal{A}$ by $\mathcal{F}_{\mathcal{A}}=\left\{F^{\text {fill }} \mid F \in \mathcal{F}_{\mathcal{B}}\right\}$. We denote fibres of $\mathcal{F}_{\mathcal{A}}$ by $\hat{F}$, and given $z \in \mathcal{A}$ we let $\widehat{F}(z)$ be the unique fibre in $\mathcal{F}_{\mathcal{A}}$ which contains $z$. Note that $F(z)=\partial \hat{F}(z)$, and so $\widehat{F}(z)=(\partial \hat{F}(z))^{\text {fill }}$.

Remark 5.5. An alternative way to define the fibres of $\mathcal{A}$ is the following. Recall that we write $C<z$ for a cut $C$ and $z \in \mathcal{A}$ iff $z \in \mathcal{R}(C)^{\text {fill }}$, and $z<C$ iff $z \in \mathcal{L}(C)^{\text {fill }}$. The notions $C \ll z$ and $z \ll C$ in $\mathcal{F}_{\mathcal{A}}$ can then be defined as before by the existence of uncountably many intermediate cuts. Using this, the fibres of $\mathcal{A}$ can be defined exactly in the same way as those of $\mathcal{B}$ in 5.1 . Equivalence of the two definitions is provided by the following statement. 
Lemma 5.6. For any $z \in \mathcal{A}$, we have

$$
\hat{F}(z)=\bigcap_{\substack{C^{-}, C^{+} \in \mathcal{C} \\ C^{-} \ll z \ll C^{+}}}\left[C^{-}, C^{+}\right]_{\mathcal{B}}^{\text {fill }} .
$$

Proof. If $z \in \mathcal{A} \backslash \mathcal{B}$ and $z^{\prime} \in \partial U_{z}$, then by definition $\widehat{F}(z)=\widehat{F}\left(z^{\prime}\right)$, and the intersection on the right hand side of 5.2 coincides as well. Thus, we may assume $z \in \mathcal{B}$. However, in this case the right side is just $F(z)^{\text {fill }}=\widehat{F}(z)$ by Lemma 5.4

Given $F^{-}, F^{+} \in \mathcal{F}_{\mathcal{B}}$, we let $\left(F^{-}, F^{+}\right)_{\mathcal{B}}=\bigcup_{F^{-}<F<F^{+}} F=\left\{z \in \mathcal{B} \mid F^{-} \prec z<F^{+}\right\}$ and $\left[F^{-}, F^{+}\right]_{\mathcal{B}}=F^{-} \cup\left(F^{-}, F^{+}\right)_{\mathcal{B}} \cup F^{+}$. Note that these intervals are relatively open, respectively relatively closed, in $\mathcal{B}$. If $\widehat{F}$ and $\widehat{F}^{\prime}$ are distinct fibres in $\mathcal{F}_{\mathcal{A}}$, then $F=\partial \widehat{F}$ and $F^{\prime}=\partial F^{\prime}$ are fibres in $\mathcal{F}_{\mathcal{B}}$. According to Lemma 5.3, we always have either $F \prec F^{\prime}$ or $F^{\prime}<F$, so either $\widehat{F} \prec \hat{F}^{\prime}$ or $\hat{F}^{\prime}<\widehat{F}$, and the notions $<$ and $\ll$ coincide for the pairs $\widehat{F}, \widehat{F}^{\prime}$ and $F, F^{\prime}$. As before, given $\widehat{F}^{-} \prec \widehat{F}^{+}$, we let $\left(\widehat{F}^{-}, \widehat{F}^{+}\right)_{\mathcal{A}}=\bigcup_{\hat{F}^{-}<\hat{F}<\hat{F}^{+}} \hat{F}$ and $\left[\hat{F}^{-}, \hat{F}^{+}\right]_{\mathcal{A}}=\hat{F}^{-} \cup\left(\hat{F}^{-}, \hat{F}^{+}\right)_{\mathcal{A}} \cup \hat{F}^{+}$. As a consequence of Lemma 5.4 we obtain that $\left(\widehat{F}^{-}, \widehat{F}^{+}\right)_{\mathcal{A}}=\left(\partial \widehat{F}^{-}, \partial \widehat{F}^{+}\right)_{\mathcal{B}}^{\text {fill }}$ and $\left[\widehat{F}^{-}, \widehat{F}^{+}\right]_{\mathcal{A}}=\left[\partial \widehat{F}^{-}, \partial \widehat{F}^{+}\right]_{\mathcal{B}}^{\text {fill }}$. In particular, this implies the following observation, which we state for further use.

Lemma 5.7. Given $F^{-}, F^{+}$, we have that $\left(\widehat{F}^{-}, \widehat{F}^{+}\right)_{\mathcal{A}}$ is relatively open and $\left[\widehat{F}^{-}, \widehat{F}^{+}\right]_{\mathcal{A}}$ is relatively closed in $\mathcal{A}$.

Moreover, we have

Lemma 5.8. For all $\widehat{F}^{-} \prec \widehat{F}^{+} \in \mathcal{F}_{\mathcal{A}}$, the set $\left[\widehat{F}^{-}, \widehat{F}^{+}\right]_{\mathcal{A}}$ is a non-separating continuum. Proof. If $\widehat{F}^{-}=\hat{F}\left(z^{-}\right)$and $\hat{F}^{+}=\widehat{F}\left(z^{+}\right)$with $z^{-}, z^{+} \in \mathcal{B}$, then

$$
\partial\left[\hat{F}^{-}, \hat{F}^{+}\right]_{\mathcal{A}}=\bigcap_{\substack{C \\ z^{+} \ll z^{-}}}\left[C^{-}, C^{+}\right]_{\mathcal{B}} .
$$

Hence, as a nested intersection of continua the set $\partial\left[\widehat{F}^{-}, \widehat{F}^{+}\right]_{\mathcal{B}}$ is connected, and so $\left[\widehat{F}^{-}, \hat{F}^{+}\right]_{\mathcal{A}}=\left(\partial\left[\hat{F}^{-}, \hat{F}^{+}\right]_{\mathcal{B}}\right)^{\text {fill }}$ is a filled - and hence non-separating - continuum.

Lemma 5.9. $\mathcal{F}_{\mathcal{A}}$ is an upper semicontinuous decomposition of $\mathcal{A}$.

Proof. Let $U \subseteq \mathcal{A}$ be an open set. We need to show that $V=\{z \in \mathcal{A}: \hat{F}(z) \subseteq U\}$ is open in $\mathcal{A}$. Let $z \in V$, so that $\hat{F}(z) \subseteq U$. From Lemma 5.6 it is easy to verify that $\widehat{F}(z)$ is the intersection of all sets of the form $\left[\widehat{F}^{-}, \widehat{F}^{+}\right]_{\mathcal{A}}$ with $\vec{F}^{-}, \widehat{F}^{+} \in \mathcal{F}_{\mathcal{A}}$ and $\hat{F}^{-} \ll \hat{F}(z) \ll \hat{F}^{+}$, and since this can be seen as a decreasing intersection, such $\widehat{F}^{+}$and $\hat{F}^{-}$may be chosen satisfying $\left[\widehat{F}^{-}, \widehat{F}^{+}\right]_{\mathcal{A}} \subset U$. Since $\left(\widehat{F}^{-}, \widehat{F}^{+}\right)_{\mathcal{A}}$ is open in $\mathcal{A}$ and contains $z \in V$, which was chosen arbitrarily, it follows that $V$ is open in $\mathcal{A}$ as claimed.

Lemma 5.10. $\mathcal{F}_{\mathcal{A}}$ projects to an upper semicontinuous decomposition $\mathcal{F}_{A}=\{\pi(F) \mid F \in$ $\left.\mathcal{F}_{\mathcal{A}}\right\}$ of $A$ into cellular continua, and it has uncountably many elements.

Proof. Since by hypothesis there exists a self-homeomorphism $\varphi$ of $\mathbb{A}$ leaving $A$ invariant without periodic points in $A$, and we may assume that $\varphi$ is orientation-preserving (replacing it by $\varphi^{2}$ if necessary), Theorem 1.1 yields the existence of a monotone map $h: A \rightarrow \mathbb{T}^{1}$, which lifts to a monotone map $H: \mathcal{A} \rightarrow \mathbb{R}$ that commutes with the translation $T$. It is easily checked that for every $x \in \mathbb{R}$ the set $G_{x}=H^{-1}([x, x+1])$ is a generator of $\mathcal{A}$, and consequently every fibre $H^{-1}(x)=T^{-1}\left(G_{x}\right) \cap G_{x}$ contains a cut. This further implies that every fibre of $H$ contains (at least) one element of $\mathcal{F}_{\mathcal{A}}$.

In particular, the above yields that $T^{k} F \cap F=\varnothing$ for all $F \in \mathcal{F}_{\mathcal{A}}$ and $k \in \mathbb{Z} \backslash\{0\}$, hence $\mathcal{F}_{\mathcal{A}}$ projects to a decomposition of $A$ with uncountably many elements, and each $F \in \mathcal{F}_{\mathcal{A}}$ projects injectively into $A$. Since $F$ is a cellular continuum, this implies that $\pi(F)$ is a cellular continuum as well. Upper semicontinuity of $\mathcal{F}_{A}$ then follows directly from that of $\mathcal{F}_{\mathcal{A}}$ and from the fact that $\pi$ is an open map: if $U \subset A$ is a neighborhood of $F \in \mathcal{F}_{A}$ in $A$, then the set of all elements of $\mathcal{F}_{A}$ contained in $U$ is the projection of the set of elements of $\mathcal{F}_{\mathcal{A}}$ contained in the open set $\pi^{-1}(U) \subset \mathcal{A}$, and therefore is open in $A$.

Let $\mathcal{F}$ be the decomposition of $A$ consisting of all elements of $\mathcal{F}_{A}$ together with all sets of the form $\{z\}$ with $z \notin A$. Note that $\mathcal{F}$ is a Moore decomposition of $\mathcal{A}$, and therefore Theorem 2.3 applies. 
Proposition 5.11. The Moore projection $\Pi: \mathbb{A} \rightarrow \mathbb{A}$ provided by Theorem 2.3 maps $A$ to an essential simple closed curve.

Proof. Due to Lemma 2.5 it suffices to show that if $x, y \in \Pi(A)$ are different points, then $\Pi(A) \backslash\{x, y\}$ is disconnected. By Lemma 2.2 this is the same as saying that for any pair of distinct elements $F_{1}, F_{2}$ of $\mathcal{F}_{A}$, the set $A \backslash\left(F_{1} \cup F_{2}\right)$ is disconnected. To show this, let $\hat{F}_{1}, \hat{F}_{2} \in \mathcal{F}_{\mathcal{A}}$ be connected components of $\Pi^{-1}\left(F_{1}\right)$ and $\Pi^{-1}\left(F_{2}\right)$. Note that since $T^{-1} F \ll F \ll T F$ for all $F \in \mathcal{F}_{\mathcal{A}}$, the projection $\Pi$ is injective on $\left(\widehat{F}_{1}, T \widehat{F}_{1}\right)_{\mathcal{A}}$, and there is exactly one $k \in \mathbb{Z}$ such that $\widehat{F}_{1} \ll T^{k} \widehat{F}_{2} \ll T \widehat{F}_{1}$. If $U=\left(\widehat{F}_{1}, T^{k} \widehat{F}_{2}\right)_{\mathcal{A}}$ and $V=\left(T^{k} \widehat{F}_{2}, T \widehat{F}_{1}\right)_{\mathcal{A}}$, we have that $\Pi(U)$ and $\Pi(V)$ are disjoint nonempty open subsets of $A$, and $\Pi(U) \cup \Pi(V)=A \backslash\left(F_{1} \cup F_{2}\right)$, proving that the latter set is disconnected.

Lemma 5.12. Suppose $h: A \rightarrow \mathbb{T}^{1}$ is a monotone map. Then the fibres $h^{-1}(x), x \in \mathbb{T}^{1}$, are saturated with respect to $\mathcal{F}_{A}$.

Proof. The map $h$ lifts to a monotone map $H: \mathcal{A} \rightarrow \mathbb{R}$ that commutes with the deck translation $T$. As argued in the proof of Lemma 5.10 every fibre $H^{-1}(x)$ contains at least one cut. If $z \in H^{-1}(x) \cap \mathcal{B}$ and $z^{\prime} \in H^{-1}(y) \cap \mathcal{B}$ with $x<y$, this implies that $z \ll z^{\prime}$ and thus $F(z) \neq F\left(z^{\prime}\right)$. Hence, no two points of the same fibre of $\mathcal{F}_{\mathcal{B}}$ can be contained in different fibres of $H$. In other words, the fibres of $H_{\mid \mathcal{B}}$ are saturated with respect to $\mathcal{F}_{\mathcal{B}}$.

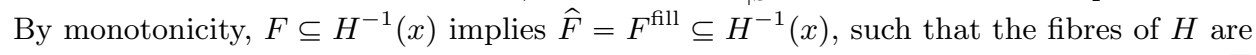
also saturated with respect to $\mathcal{F}_{A}$.

5.1 Proof of Theorem 1.2 Denote by $\Pi: \mathbb{A} \rightarrow \mathbb{A}$ the Moore projection associated to $\mathcal{F}_{\mathcal{A}} \cup\{x \in \mathbb{A} \mid x \notin A\}$, so it satisfies assertion (i) of Theorem 1.2 . Assertions (ii) and (iii) follow from Proposition 5.11 and Schönflies' theorem (by post-composing with a homeomorphism of $\mathbb{A}$ which maps $\Pi(A)$ to $\mathbb{T}$ ). Since the lift $\Phi$ of any homeomorphism $\varphi$ of $\mathbb{A}$ leaving $A$ invariant has to map minimal generators to minimal generators, it follows from the above constructions that $\Phi$ permutes the elements of $\mathcal{F}_{\mathcal{A}}$. This allows to define $\tilde{\varphi}: \mathbb{A} \rightarrow \mathbb{A}$ by requiring that $\Pi^{-1}(\tilde{\varphi}(z))=\varphi\left(\Pi^{-1}(z)\right)$. If $U \subseteq \mathbb{A}$ is open, then $\tilde{\varphi}^{-1}(U)=$ $\Pi\left(\varphi^{-1}\left(\Pi^{-1}(U)\right)\right.$ is open as well, since $\Pi$ maps saturated open sets to open sets. Hence, $\tilde{\varphi}$ is continuous and assertion (iv) holds. To prove assertion (v), suppose that $h: A \rightarrow \mathbb{T}^{1}$ is a monotone surjection, then by Lemma $5.12 h^{-1}(x)$ is saturated with respect to $\mathcal{F}_{A}$, and thus $h$ induces a map $\tilde{h}: \mathbb{T} \rightarrow \mathbb{T}^{1}$ by $\tilde{h}(x)=h\left(x^{\prime}\right)$ where $x^{\prime} \in \Pi^{-1}(x)$ is arbitrary. Note that $\widetilde{h}$ is continuous since for any open set $U \subset \mathbb{T}^{1}$ we have $\widetilde{h}^{-1}(U)=\Pi\left(h^{-1}(U)\right)$ which is again open since it is the image of a saturated open set. If $h$ is a semiconjugation from $\varphi$ to $R_{\rho}$, then given $x \in \mathbb{T}^{1}$ and $x^{\prime} \in \Pi^{-1}(x)$ we have

$$
\tilde{h} \circ \tilde{\varphi}(x)=\tilde{h} \circ \tilde{\varphi} \circ \Pi(z)=\tilde{h} \circ \Pi \circ \varphi(z)=h \circ \varphi(z)=R_{\rho} \circ h(z)=R_{\rho} \circ \tilde{h}(x) .
$$

completing the proof if $(\mathrm{v})$.

5.2 Proof of Corollary $\mathbf{1 . 3}$ Suppose $h_{1}, h_{2}$ are two semiconjugacies from $\varphi_{\mid A}$ to the irrational rotation $R_{\rho}$, where $\rho$ is the rotation number of $\varphi$ on $A$. By post-composing with a rotation, we may assume that there exists $z_{0} \in A$ with $h_{1}\left(z_{0}\right)=h_{2}\left(z_{0}\right)$.

Let $\tilde{\varphi}$ be the homeomorphism of $\mathbb{A}$ from part (iv) of Theorem 1.2 so that $\Pi \circ \varphi=\tilde{\varphi} \circ \Pi$ and $\tilde{\varphi}(\mathbb{T})=\mathbb{T}$, and let $\widetilde{h}_{i}$ the maps such that $h_{i}=\tilde{h} \circ \Pi$ from part (v) of the same theorem. Then $\widetilde{h}_{i}$ semiconjugates $\left.\widetilde{\varphi}\right|_{\mathbb{T}}$ to the irrational rotation $R_{\rho}$ of $\mathbb{T}$. Since the semiconjugacies in the Poincaré Classification Theorem are unique up to post-composition by a rotation, this yields that $\tilde{h}_{1}=\tilde{h}_{2}$ and thus $h_{1}=h_{2}$.

\section{Almost automorphic minimal continua}

A homeomorphism $f: X \rightarrow X$ of a metric space is called almost automorphic if there exists $x \in X$ such that, whenever the limit $\tilde{x}=\lim _{k \rightarrow \infty} f^{n_{k}}(x)$ exists for some sequence $n_{k} \rightarrow \infty$, then $x=\lim _{k \rightarrow \infty} f^{-n_{k}}(\widetilde{x})$. Further, $f$ is almost periodic if for every $\epsilon>0$, the set $\left\{n \in \mathbb{Z}: \forall x \in X, d\left(f^{n}(x), x\right)<\epsilon\right\}$ is syndetic (i.e. has uniformly bounded gaps). The Veech Structure Theorem Vee65] asserts that $f$ is almost automorphic if and only if $f$ is semiconjugate to an almost periodic map of some space $Y$ by means of an almost 1-1 continuous surjection, i.e. a continuous surjection $h: X \rightarrow Y$ for which the set of points of $Y$ with a unique preimage is dense. 
6.1 Proof of Theorem 1.4 Let $\varphi: \mathbb{A} \rightarrow \mathbb{A}$ be a homeomorphism, $B \subset \mathbb{A}$ is an essential $\varphi$-invariant cobasin boundary without periodic points, and $x_{0} \in B$ a recurrent bi-accessible point. Since $x_{1}=\varphi\left(x_{0}\right)$ is also bi-accessible, we may find an inessential simple closed curve $\gamma \subset \mathbb{A}$ intersecting $B$ exactly at $x_{0}$ and $x_{1}$. If $V_{0}$ and $V_{1}$ are the two connected components of $\mathbb{A} \backslash \gamma$ and $B_{i}=V_{i} \cap B$, it is easy to verify that $B_{i} \cup\left\{x_{0}, x_{1}\right\}$ is a continuum for $i \in\{0,1\}$. Thus $B$ is decomposable, and Theorem 1.2 implies that there exists a $\Pi: \mathbb{A} \rightarrow \mathbb{A}$ mapping the circloid $A$ whose boundary is $B$ to the circle $\mathbb{T}=\mathbb{T}^{1} \times\{0\}$ and inducing a map $\tilde{\varphi}: \mathbb{A} \rightarrow \mathbb{A}$ which satisfies $\tilde{\varphi} \circ \Pi=\Pi \circ \varphi$ and preserves $\mathbb{T}$. Let $U^{-}$ and $U^{+}$denote the connected components of $\mathbb{A} \backslash B$ which are unbounded below and above, respectively, and let $C_{\widetilde{C}}$ be a simple arc joining a point of $U^{-}$to a point of $U^{+}$such that $C \cap B=\left\{x_{0}\right\}$. Then $\widetilde{C}=\Pi(C)$ is an arc joining a point below $\mathbb{T}$ to a point above $\mathbb{T}$ and intersecting $\mathbb{T}$ exactly at $\widetilde{x}_{0}=\Pi\left(x_{0}\right)$. By continuity, the compact set $\Pi^{-1}(\widetilde{C})$ is contained in the closure of $\Pi^{-1}\left(\widetilde{C} \backslash\left\{\widetilde{x}_{0}\right\}\right)$, and since $\left.\Pi\right|_{U^{-} \cup U^{+}}$is an injective map onto $\mathbb{A} \backslash \mathbb{T}$ we have that $\Pi^{-1}\left(\widetilde{C} \backslash\left\{\widetilde{x}_{0}\right\}\right)=C \backslash\left\{x_{0}\right\}$. Thus $\Pi^{-1}(\widetilde{C}) \subset C$, and $\Pi^{-1}\left(\widetilde{x}_{0}\right) \subset C \cap B=\left\{x_{0}\right\}$.

Hence, $\Pi^{-1}\left(\Pi\left(x_{0}\right)\right)=\left\{x_{0}\right\}$, and the same is true for any point in the orbit of $x_{0}$. Since $\tilde{\varphi}: \mathbb{T} \rightarrow \mathbb{T}$ is a homeomorphism of the circle without periodic points, the classic Poincaré theory implies that it is semi-conjugate to an irrational rotation $R_{\rho}$ of the circle by means of a continuous monotone surjection $h: \mathbb{T} \rightarrow \mathbb{T}^{1}$ which is injective in the nonwandering set of $\tilde{\varphi}$. In particular, since $\widetilde{x}_{0}$ is recurrent, $h^{-1}\left(h\left(\widetilde{x}_{0}\right)\right)=\left\{\widetilde{x}_{0}\right\}$, and so $h^{\prime}=h \circ \Pi$ is an almost 1-1 semiconjugation between $\left.\varphi\right|_{B}$ and $R_{\rho}$, showing that $\left.\varphi\right|_{B}$ is almost-automorphic.

6.2 Proof of Corollary 1.5 Let $U^{-}$and $U^{+}$be the connected components of $\mathbb{A} \backslash X$ unbounded below and above, respectively. Then $\partial U^{-} \cup \partial U^{+}$is a compact invariant subset of $X$ and therefore is equal to $X$. In particular $\partial U^{-} \cap \partial U^{+} \neq \varnothing$, and since that is also a compact invariant set we deduce $X=\partial U^{-} \cap \partial U^{+}$. Therefore, $X$ is a cofrontier, and the Corollary follows from Theorem 1.4

\section{The Kuratowski decomposition}

Let $\Lambda$ be a continuum on the sphere $\mathbb{S}^{2}$ which is the common boundary of two open simply connected sets. Using our terminology, this is the same as saying that $\Lambda$ is a cobasin boundary.

In Kur28, Kuratowski studied the following topological problem: when can we find a monotone surjection from $\Lambda$ onto the circle? Following the method used in [Kur27] to study a similar question for irreducible continua, he defined a decomposition into layer $\$^{3}$ as follows: A fundamental layer of $\Lambda$ is any subset of $\Lambda$ which is maximal with the property of being a continuum which is the union of at most countably many subcontinua of $\Lambda$, each of which either is indecomposable or has empty interior in $\Lambda$. When there is a unique fundamental layer, the continuum $\Lambda$ is called monostratic.

The main results of Kur28] implies that $\Lambda$ is non-monostratic if and only if there exists some monotone continuous surjection from $\Lambda$ onto the circle. In fact, if $\Lambda$ is nonmonostratic then the fundamental layers form a monotone upper semicontinuous decomposition of $\Lambda$, and the quotient space by this decomposition is a simple closed curve. In addition, the decomposition into fundamental layers is the finest upper semicontinuous monotone decomposition of $\Lambda$ into subcontinua with the property of having the circle as a quotient space, in the sense that any other such decomposition has its elements saturated by fundamental layers.

This can be stated in terms of maps as follows:

Theorem 7.1 (Kuratowski). If $\Lambda$ is a non-monostratic cobasin boundary in $\mathbb{S}^{2}$, then there exists a monotone continuous surjection $\Pi: \Lambda \rightarrow \mathbb{T}^{1}$ such that for any other monotone continuous surjection $P: \Lambda \rightarrow \mathbb{T}^{1}$, there exists a map $\phi: \Lambda \rightarrow \Lambda$ such that $P=\phi \circ \Pi$.

There are many works which extend to a more general setting the concept of finding a finest monotone upper semicontinuous decomposition with the property of having a quotient space with a given property (what is often called the core decomposition with the given property); see for instance Cha73, FS67, Rak77, Vou74] and references therein.

Using the previous theorem together with Moore's theorem, Kuratowski obtains a result which can be reworded as follows [Kur28, Theorem II]:

\footnotetext{
${ }^{3}$ Translation of the original term tranches (in french).
} 
Theorem 7.2. If $\Lambda$ is a circloid in $\mathbb{S}^{2}$ with non-monostratic boundary, then there exists a monotone continuous surjection $\Pi: \mathbb{S}^{2} \rightarrow \mathbb{S}^{2}$ which maps $\Lambda$ onto the equator and is injective on $\mathbb{S}^{2} \backslash \Lambda$.

In fact, it can easily be verified that the decomposition of $\Lambda$ into fibers of $\Pi$ is the core decomposition with respect of having the circle as quotient space. The map $\Pi$ can be characterized by considering the decomposition of $\mathbb{S}^{2}$ into points of $\mathbb{S}^{2} \backslash \Lambda$ together with the "filled" layers of $\partial \Lambda$ (i.e. the union of each layer $L$ with all the connected components of $\mathbb{S}^{2} \backslash L$ which are disjoint from $\Lambda$ ). This decomposition turns out to be the same given in the proof of Theorem 1.2

In the proof of Theorem 1.2 , we could have used Theorems 7.1 and 7.2 , but we chose a self-contained proof. We note that the only part of Section 5 where dynamics appears is to guarantee that there are uncountably many elements the decomposition $\mathcal{F}_{A}$ (Lemma 5.10 . Since it can be shown without too much effort that uncountability of $\mathcal{F}_{\mathcal{A}}$ is equivalent to non-monostraticity, the results of Section 5 essentially contain an alternative proof of Kuratowski's results (if one assumes non-monostraticity instead of the dynamical hypothesis).

In this context, the main contribution of Theorem 1.1 could also be stated as follows: if a circloid is invariant by a homeomorphism, has decomposable boundary and has no periodic points, then its boundary is non-monostratic.

\section{An example with large fibers}

In this section we prove Theorem 1.6. More specifically, we will prove that there exists a $C^{\infty}$ diffeomorphism $f: \mathbb{A} \rightarrow \mathbb{A}$ and a monotone continuous surjection $\Pi: \mathbb{A} \rightarrow \mathbb{A}$ such that:

- $f$ leaves invariant an essential decomposable cofrontier $\Lambda$;

- $\left.f\right|_{\Lambda}$ is minimal;

- $\Pi$ semi-conjugates $f$ with an irrational rotation of $\mathbb{A}$ : $\Pi f=R_{\rho} \Pi$ for some $\rho \notin \mathbb{Q}$;

- Each fiber of $\left.\Pi\right|_{\Lambda}$ is a continuum with diameter at least $1 / 4$.

In what follows, intervals in $\mathbb{T}^{1}$ are assumed to be positively oriented, so for $a, b \in \mathbb{T}^{1}$ the interval $(a, b)$ is the component of $\mathbb{T}^{1} \backslash\{a, b\}$ which is positively oriented and $(b, a)$ the remaining one (and similarly for closed intervals).

8.1 The Anosov-Katok method We will use the Anosov-Katok method, which we describe here briefly (and refer to FK04 for more details and further references). The map $f$ will be obtained as a limit of maps $f_{n}$, each of which is $C^{\infty}$-conjugate to a rational rotation $R_{p_{n} / q_{n}}:(\theta, y) \mapsto\left(\theta+p_{n} / q_{n}, y\right)$, so

$$
f_{n}=H_{n} R_{p_{n} / q_{n}} H_{n}^{-1}
$$

where $H_{n} \in \operatorname{Diff}^{\infty}(\mathbb{A})$. The maps $H_{n}$ are successive compositions of maps:

$$
H_{n}=h_{1} \circ \cdots \circ h_{n-1} \circ h_{n} .
$$

The maps $h_{n} \in \operatorname{Diff}^{\infty}(\mathbb{A})$ are chosen inductively alongside with the numbers $p_{n} / q_{n}$ with the following condition: If $h_{n}$ and $p_{n} / q_{n}$ is already chosen, $h_{n+1}$ can be chosen arbitrarily, with the only restriction that it commutes with $R_{p_{n} / q_{n}}$ :

$$
h_{n+1} R \frac{p_{n}}{q_{n}}=R \frac{p_{n}}{q_{n}} h_{n+1}
$$

This guarantees that for any $\tau$,

$$
H_{n+1} R_{\tau} H_{n+1}^{-1}=H_{n} h_{n+1} R_{\tau} h_{n+1}^{-1} H_{n}^{-1}=H_{n}\left(h_{n+1} R_{\left(\tau-\frac{p_{n}}{q_{n}}\right)} h_{n+1}^{-1}\right) R \frac{p_{n}}{q_{n}} H_{n}^{-1},
$$

which means that if $\tau=p_{n+1} / q_{n+1}$ is chosen close enough to $p_{n} / q_{n}$ (but different from it), the map $f_{n+1}$ can be made arbitrarily $C^{\infty}$-close to $f_{n}$, and in particular one can make the $C^{n}$-distance $\xi_{n}=d_{C^{n}}\left(f_{n+1}, f_{n}\right)+d_{C^{n}}\left(f_{n+1}^{-1}, f_{n}^{-1}\right)$ as small as desired. Repeating this process, if the numbers $\xi_{n}$ are chosen such that $\sum_{n \in \mathbb{N}} \xi_{n}<\infty$, the maps $f_{n}$ converge in the $C^{\infty}$-topology to an element of $\operatorname{Diff}^{\infty}(\mathbb{A})$. 
Since there is a great degree of freedom in the choice of $h_{n}$ as well as $p_{n} / q_{n}$ at each step, additional restrictions may be placed in order to guarantee that the limit map has special properties. To outline the construction that we will follow, our choice of these maps will be such that there exists a decreasing sequence of essential annuli $A_{n}$ such that $h_{n+1}$ is the identity outside $A_{n}$ and maps $A_{n+1}$ into $A_{n}$ in such a way that every horizontal circle in $A_{n+1}$ becomes $\epsilon$-dense in $A_{n}$ (with an appropriate choice of $\epsilon$ depending on $n$ ) and every vertical segment in $A_{n+1}$ is mapped to a set with diameter greater than $1 / 4$. This is achieved by first using a map that "twists" vertical segments inside $A_{n+1}$ so that any such segment becomes horizontally large, and then composing with a map that maps $A_{n+1}$ to something that oscillates vertically inside $A_{n}$ (see Figure 8.1.


Figure 8.1: How $h_{n+1}$ maps a vertical segment in $A_{n+1}$ (in two steps).

The cofrontier $\Lambda$ will be the intersection of the sets $\Lambda_{n}=H_{n}\left(A_{n}\right)$, which is a decreasing intersection. An appropriate choice of $p_{n+1} / q_{n+1}$ and the fact that $h_{n+1}$ spreads $\epsilon$-densely in $A_{n}$ every horizontal circle will guarantee that $\left.f\right|_{\Lambda}$ is minimal and $p_{n} / q_{n}$ converges to an irrational number $\alpha$. Moreover, although the maps $H_{n}$ are not required to converge to a homeomorphism, we will guarantee that $H_{n}^{-1}$ does converge to a continuous surjection $\Pi$ in the $C^{0}$ topology. Due to the way in which these maps are defined, this automatically implies that $\Pi$ semi-conjugates $f$ with the rotation $R_{\alpha}$, and the conditions on $h_{n}$ will guarantee that the preimage of any point of $\Lambda$ by $\Pi$ has diameter at least $1 / 4$.

Let $\left(\alpha_{n}\right)_{n \in \mathbb{N}}$ and $\left(\epsilon_{n}\right)_{n \in \mathbb{N}}$ be decreasing sequences of positive real numbers such that $\alpha_{1}<1, \alpha_{n} \rightarrow 0$ as $n \rightarrow \infty$, and $\sum_{n \in \mathbb{N}} \epsilon_{n}<\infty$.

Claim 8.1. There exist sequences $\left(h_{n}\right)_{n \in \mathbb{N}}$ in $\operatorname{Diff}^{\infty}(\mathbb{A}),\left(p_{n} / q_{n}\right)_{n \in \mathbb{N}}$ in $\mathbb{Q}$ (where $p_{n}, q_{n}$ are relatively prime integers, $\left.q_{n}>0\right)$, and $\left(M_{n}\right)_{n \in \mathbb{N}}$ in $\mathbb{N}$ such that, letting

- $H_{n}=h_{1} h_{2} \cdots h_{n}$,

- $f_{n}=H_{n} R_{p_{n} / q_{n}} H_{n}^{-1}$,

- $A_{n}=\mathbb{T}^{1} \times\left[-\alpha_{n}, \alpha_{n}\right]$,

- $\Lambda_{n}=H_{n}\left(A_{n}\right)$,

the following properties hold for $k \geqslant 1$ :

(1) $h_{k}\left(A_{k}\right)=A_{k}$, and $h_{k}(z)=z$ for all $z \in \mathbb{A} \backslash A_{k}$;

(2) $\operatorname{diam}\left(H_{k}\left(\{\theta\} \times\left[-\alpha_{k}, \alpha_{k}\right]\right)\right)>1 / 4$ for all $\theta \in \mathbb{T}^{1}$;

(3) $q_{k}>13^{k}$;

and for $k \geqslant 2$,

(4) $h_{k} R_{p_{k-1} / q_{k-1}}=R_{p_{k-1} / q_{k-1}} h_{k}$;

(5) $H_{k}\left(\mathbb{T}^{1} \times\{\alpha\}\right)$ is $\epsilon_{k-1}$-dense in $\Lambda_{k-1}$ for all $\alpha \in\left[-\alpha_{k}, \alpha_{k}\right]$;

(6) $d\left(\pi_{1}\left(h_{k}(z)\right), \pi_{1}(z)\right)<3 / q_{k-1}$ for all $z \in \mathbb{A}$;

(7) $d_{C^{k}}\left(f_{k}, f_{k-1}\right)<\epsilon_{k-1}$ and $d_{C^{k}}\left(f_{k}^{-1}, f_{k-1}^{-1}\right)<\epsilon_{k-1}$;

(8) $\left|p_{k} / q_{k}-p_{k-1} / q_{k-1}\right|<\epsilon_{k-1}$;

(9) $\left\{f_{k}^{j}(z): 0 \leqslant j \leqslant M_{i+1}\right\}$ is $\epsilon_{i}$-dense in $\Lambda_{i}$ for all $z \in \Lambda_{k}$ and $1 \leqslant i \leqslant k-1$; 
Before explaining how to obtain such sequences, let us prove that they lead to a map with the required properties.

Claim 8.2. Using the maps defined in Claim 8.1, there exist $f \in \operatorname{Diff}^{\infty}(\mathbb{A}), \Pi \in C^{0}(\mathbb{A}, \mathbb{A})$, and $\rho \in \mathbb{T}^{1}$ such that, if $\Lambda=\bigcap_{n \in \mathbb{N}} \Lambda_{n}$, then

- $f_{n} \rightarrow f$ in the $C^{\infty}$ topology as $n \rightarrow \infty$;

- $H_{n}^{-1} \rightarrow \Pi$ in the $C^{0}$ topology as $n \rightarrow \infty$;

- $\Pi$ is a monotone semiconjugation between $f$ and $R_{\rho}$;

- $\Lambda$ is an essential $f$-invariant decomposable cofrontier and $\Pi(\Lambda)=\mathbb{T}^{1} \times\{0\}$;

- $\operatorname{diam}\left(\Pi^{-1}(\Pi(z))\right) \geqslant 1 / 4$ for all $z \in \Lambda$, and $\left.\Pi\right|_{\mathbb{A} \backslash \Lambda}$ is injective;

- $\left.f\right|_{\Lambda}$ is minimal.

Proof. Note that

$$
d_{C^{0}}\left(H_{n}^{-1}, H_{n+m}^{-1}\right)=d_{C^{0}}\left(H_{q}^{-1} n H_{n+m}, H_{n+m}^{-1} H_{n+m}\right)=d_{C^{0}}\left(h_{n+1} h_{n+2} \cdots h_{n+m}, \text { Id }\right) .
$$

By (6) one has

$$
\left|\pi_{1}\left(h_{n+1} h_{n+2} \cdots h_{n+m}(z)\right)-\pi_{1}(z)\right| \leqslant \sum_{i=n}^{n+m-1} \frac{3}{q_{i}},
$$

and by (1) since each $h_{n+i}$ leaves $A_{n}$ invariant and is the identity outside $A_{n}$,

$$
\left|\pi_{2} h_{n+1} h_{n+2} \cdots h_{n+m}(z)-\pi_{2}(z)\right| \leqslant 2 \alpha_{n} .
$$

Since $\alpha_{n} \rightarrow 0$ and $\sum_{i} 1 / q_{i}<\infty$, we see that the two coordinates of the map

$$
z \mapsto h_{n+1} h_{n+2} \cdots h_{n+m}(z)-z
$$

are uniformly small if $n, m$ are large enough, and therefore $\left(H_{i}^{-1}\right)_{i \in \mathbb{N}}$ is a Cauchy sequence in $C^{0}(\mathbb{A}, \mathbb{A})$, so it converges to some continuous (and surjective) map $\Pi$. Note also that $H_{i}^{-1}(z)$ is eventually constant if $z \notin \Lambda$. Since each $H_{i}^{-1}$ is a homeomorphism onto its image, it follows easily that $\left.\Pi\right|_{\mathbb{A} \backslash \Lambda}$ is injective, and $\Pi(\Lambda)=\mathbb{T}^{1} \times\{0\}$.

By (7) and the fact that $\sum_{n} \epsilon_{n}<\infty$, the sequences $\left(f_{n}\right)_{n \in \mathbb{N}}$ and $\left(f_{n}^{-1}\right)_{n \in \mathbb{N}}$ are Cauchy in the $C^{r}$-topology for any $r$, the sequence $\left(f_{n}\right)_{n \in \mathbb{N}}$ converges to some $C^{\infty}$ diffeomorphism $f$ in the $C^{\infty}$-topology.

By (8) the sequence $\left(p_{n} / q_{n}\right)_{n \in \mathbb{N}}$ is Cauchy and therefore has some limit $\rho \in \mathbb{T}^{1}$. oreover, since

$$
H_{n}^{-1} f_{n}=H_{n}^{-1}\left(H_{n} R_{p_{n} / q_{n}} H_{n}^{-1}\right)=R_{p_{n} / q_{n}} H_{n}^{-1}
$$

taking limits as $n \rightarrow \infty$ one deduces that $\Pi f=R_{\rho} \Pi$. The fact that $\Pi$ is a uniform limit of homeomorphisms implies that $\Pi$ is monotone (see for instance Why55]), so the preimages of points are connected. Moreover, note that $H_{n}\left(\{\theta\} \times\left[-\alpha_{n}, \alpha_{n}\right]\right)$ is connected and has diameter at least $1 / 4$ due to (2) Choosing $z_{n}, z_{n}^{\prime} \in H_{n}\left(\{\theta\} \times\left[-\alpha_{n}, \alpha_{n}\right]\right)$ such that $d\left(z_{n}, z_{n}^{\prime}\right) \geqslant 1 / 4$ and taking convergent subsequences of $\left(z_{n}\right)$ and $\left(z_{n}^{\prime}\right)$ one finds two points $z, z^{\prime} \in \Lambda$ such that $d\left(z, z^{\prime}\right) \geqslant 1 / 4$ and $\Pi(z)=\Pi\left(z^{\prime}\right)=(\theta, 0)$, showing that $\operatorname{diam}\left(\Pi^{-1}(\theta, 0)\right) \geqslant 1 / 4$. This means that $\operatorname{diam}\left(\Pi^{-1}(\Pi(z))\right) \geqslant 1 / 4$ for all $z \in \Lambda$.

The minimality of $\left.f\right|_{\Lambda}$ follows immediately from (9) Since the set $\Lambda=\bigcap_{n \in \mathbb{N}} \Lambda_{n}$ is a decreasing intersection of essential closed topological annuli, it is an essential annular continuum, and using the fact that $\left.f\right|_{\Lambda}$ is minimal we deduce that $\Lambda$ is an essential cofrontier (by an argument already used in the proof of Corollary 1.5 .

To see that $\Lambda$ is decomposable, let $I_{1}=[0,1 / 2]$ and $I_{2}=[1 / 2,1]$ be the upper and lower half-circles, respectively, and let $\Lambda_{n}^{j}=H_{n}\left(I_{j} \times\left[-\alpha_{n}, \alpha_{n}\right]\right)$ for $j \in\{1,2\}$. For each $j$, let $\Lambda^{j}$ be the Hausdorff limit of some convergent subsequence of $\left(\Lambda_{n}^{j}\right)_{n \in \mathbb{N}}$. Then $\Lambda^{1}$ and $\Lambda^{2}$ are compact connected nonempty sets, and from the fact that $\Lambda_{n}=\Lambda_{n}^{1} \cup \Lambda_{n}^{2}$ it is easy to verify that $\Lambda=\Lambda^{1} \cup \Lambda^{2}$. On the other hand, note that from (3)] one has $\mu:=\sum_{i=1}^{\infty} 3 / q_{i}<3 \sum_{i=1}^{\infty} 1 / 13^{i}=1 / 4$, and $(6)$ implies that $d\left(\pi_{1}\left(H_{n}(z)\right), \pi_{1}(z)\right)<\mu$ (as noted in the beginning of the proof). Thus $\pi_{1}\left(\Lambda_{n}^{j}\right)=\pi_{1}\left(H_{n}\left(I_{j} \times\left[-\alpha_{n}, \alpha_{n}\right]\right)\right)$ is an interval of length at most $1 / 2+2 \mu$. Therefore $\pi_{1}\left(\Lambda^{j}\right)$ has length at most $1 / 2+2 \mu<1$, and since $\Lambda$ is essential this means that $\Lambda^{j} \neq \Lambda$ for $j \in\{1,2\}$, showing that $\Lambda=\Lambda^{1} \cup \Lambda^{2}$ is a decomposition of $\Lambda$ into nonempty proper subcontiua. 


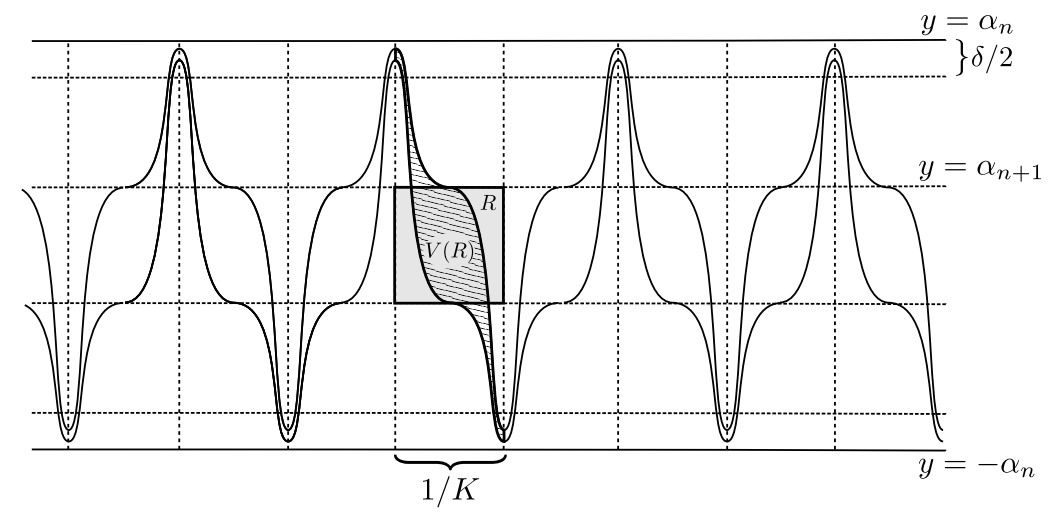

Figure 8.2: A possible map $(\theta, y) \mapsto V(\theta, y)$

Proof of Claim 8.1. We define the sequences recursively. Start with $p_{1}=0$ and $q_{1}=13^{2}$. To simplify the construction we will assume $\alpha_{1}>1 / 4$, so that letting $h_{1}=\operatorname{Id}_{\mathbb{A}}$ conditions (1) (3) automatically hold for $k=1$. Note that (9) is vacuously true when $k=1$, so we choose $M_{1}$ arbitrarily.

Assuming that $h_{k}, p_{k} / q_{k}$, and $M_{k}$ are already defined for $1 \leqslant k \leqslant n$ and satisfy the required properties, we will define them for $k=n+1$. As explained in the introduction of this section, as long as $p_{n+1} / q_{n+1}$ is chosen close enough to $p_{n} / q_{n}$ we can guarantee that $f_{n+1}$ will be arbitrarily close to $f_{n}$ in the $C^{n}$-topology. Therefore we will first choose $h_{n+1}$ accommodating to our needs, and later we will choose $p_{n+1} / q_{n+1}$ close enough to $p_{n} / q_{n}$ (but different from it) so as to guarantee conditions (7), (9) and (3).

In order to define our map $h_{n+1}$ we first introduce two auxiliary maps. Given $\delta>0$ and positive integers $K, n, q$ the first map $V=V_{n, q, \delta} \in \operatorname{Diff}^{\infty}(\mathbb{A})$ will have the following properties:

(V1) $V(z)=z$ for $z \in \mathbb{A} \backslash A_{n}$;

(V2) If $I \subset \mathbb{T}^{1}$ is any interval of length greater than $\delta$, then there is a subinterval of $I$ with endpoints $\theta_{0}, \theta_{1}$ such that $V$ maps each set $\left\{\theta_{0}\right\} \times\left[-\alpha_{n+1}, \alpha_{n+1}\right]$ and $\left\{\theta_{1}\right\} \times$ $\left[-\alpha_{n+1}, \alpha_{n+1}\right]$ into a different connected component of $A_{n} \backslash\left(\mathbb{T}^{1} \times\left[-\alpha_{n}+\delta, \alpha_{n}-\delta\right]\right)$.

(V3) $d\left(\pi_{1}(V(z)), \pi_{1}(z)\right)<\delta$ for all $z \in \mathbb{A}$.

(V4) $V$ commutes with $R_{\frac{1}{q}}$.

There are several ways to define such a map. We describe one way ${ }^{4}$, depicted in Figure 8.2 let $\phi: \mathbb{R} \rightarrow \mathbb{R}$ be a $C^{\infty}$ bump function such that $\phi(t)=1$ if $|t|<\alpha_{n+1}, \phi(t)=0$ if $|t|>\alpha_{n}$ and $0 \leqslant \phi(t) \leqslant 1$ for all $t \in \mathbb{R}$, and consider the vector field

$$
V(\theta, y)=\left(0, \sin \left(2 \pi \frac{\delta x}{4 q}\right) \phi(y)\right)
$$

on $\mathbb{A}$. If $t \in \mathbb{R}$ is large enough, the time- $t$ map of the flow induced by $V$ is a diffeomorphism satisfying the requred properties (see Figure 8.2.

The second map $T=T_{n, q} \in \operatorname{Diff}^{\infty}(\mathbb{A})$ is such that:

(T1) $T(z)=z$ for all $z \in \mathbb{A} \backslash A_{n}$ and all $z \in \mathbb{T}^{1} \times\{0\}$;

(T2) $T\left(A_{n+1}\right)=A_{n+1}$;

(T3) $[\theta-1 / q, \theta+1 / q] \subset \pi_{1}\left(T\left(\{\theta\} \times\left[-\alpha_{n+1}, \alpha_{n+1}\right]\right)\right)$ for all $\theta \in \mathbb{T}^{1}$;

(T4) $\left.d\left(\pi_{1}(T(z)), \pi_{1}(z)\right)\right) \leqslant 2 / q$ for all $z \in \mathbb{A}$;

(T5) $T$ commutes with $R_{1 / q}$.

\footnotetext{
${ }^{4}$ An alternative definition (which we will not develop further) leads to an area-preserving map $V$ with similar properties; roughly, this is done by using a map which expands $A_{n+1}$ horizontally, contracts vertically, and folds it back into $A_{n}$ while keeping all the required properties. By doing this, one could guarantee that the maps $f_{n}$ (and therefore limit map $f$ in Claim 8.2 are area-preserving.
} 
Such a map may be defined explicitly as

$$
(\theta, y) \mapsto\left(\theta+\frac{\psi(y) y}{\alpha_{n+1} q_{n}}, y\right)
$$

where $\psi: \mathbb{R} \rightarrow \mathbb{R}$ is a $C^{\infty}$ bump function such that $0 \leqslant \psi(z) \leqslant 1$ for all $z \in \mathbb{R}, \psi$ is equal to 1 on $\left[-\alpha_{n+1}, \alpha_{n+1}\right]$ and 0 outside $\left[-\alpha_{n}, \alpha_{n}\right]$ and $|\psi(y) y| \leqslant 2 \alpha_{n+1}$ for all $y \in \mathbb{R}$.

Let $s=\sup _{\theta \in \mathbb{T}^{1}} \operatorname{diam}\left(H_{n}\left(\{\theta\} \times\left[-\alpha_{n}, \alpha_{n}\right]\right)\right)-1 / 4$. By continuity of $H_{n}$ and property (2) it follows that $s>0$. Let $\epsilon=\min \left\{s, \epsilon_{n+1}\right\}$, and choose $0<\delta$ so small that $d\left(H_{n}(z), H_{n}\left(z^{\prime}\right)\right)<\epsilon / 2$ whenever $d\left(z, z^{\prime}\right)<3 \delta$, and in addition $\delta<1 / q_{n}$ and $\delta<\left(\alpha_{n}-\alpha_{n+1}\right) / 4$.

We now claim that the map $h_{n+1}=V \circ T$ satisfies the required properties using $q=q_{n}$. First, note that properties (1) and (4) for $k=n+1$ follow directly from (V1), (T1), (V4) and (T5).

Let us further note that whenever $\gamma \subset A_{n+1}$ is an arc such that $\operatorname{diam}\left(\pi_{1}(\gamma)\right)>\delta$, then $V(\gamma)$ is $3 \delta$-dense in $\pi_{1}(\gamma) \times\left[-\alpha_{n}, \alpha_{n}\right]$. Indeed, the properties of $V$ imply that $\left[-\alpha_{n}+\delta, \alpha_{n}-\delta\right] \subset \pi_{2}(V(\gamma))$ and $\pi_{1}(V(\gamma))$ lies in an $\delta$-neighborhood of $\pi(\gamma)$. Thus, given $z \in \pi_{1}(\gamma) \times\left[-\alpha_{n}+\delta, \alpha_{n}-\delta\right]$, there exists $z^{\prime} \in \gamma$ such that $\pi_{2}\left(z^{\prime}\right)=\pi_{2}(z)$ and $d\left(z^{\prime}, z\right)<2 \delta$. From this it follows easily that $V(\gamma)$ is $3 \delta$-dense in $\pi_{1}(\gamma) \times\left[-\alpha_{n}, \alpha_{n}\right]$ as claimed.

Since, for any $\alpha \in\left[-\alpha_{n+1}, \alpha_{n+1}\right]$, the set $T\left(\mathbb{T}^{1} \times\{\alpha\}\right)$ is contained in $A_{n+1}$, the previous remarks imply that $h_{n+1}\left(\mathbb{T}^{1} \times\{\alpha\}\right)=V\left(T\left(\mathbb{T}^{1} \times\{\alpha\}\right)\right)$ is $3 \delta$-dense in $A_{n}$, anqd since $H_{n+1}\left(\mathbb{T}^{1} \times\{\alpha\}\right)=H_{n}\left(h_{n+1}\left(\mathbb{T}^{1} \times\{\alpha\}\right)\right)$, the choice of $\delta$ implies that $H_{n+1}\left(\mathbb{T}^{1} \times\{\alpha\}\right)$ is $\epsilon$-dense in $H_{n}\left(A_{n}\right)=\Lambda_{n}$. Since $\epsilon<\epsilon_{n+1}$, this shows that property (5) holds for $k=n+1$.

Given $\theta \in \mathbb{T}^{1}$, let $\gamma=T\left(\{\theta\} \times\left[-\alpha_{n+1}, \alpha_{n+1}\right]\right)$. Then $\left[\theta-1 / q_{n}, \theta+1 / q_{n}\right] \subset \pi_{1}(\gamma)$, and in particular $\operatorname{diam}\left(\pi_{1}(\gamma)\right)>2 / q_{n}>\delta$, so that $V(\gamma)$ is $3 \delta$-dense in $\pi_{1}(\gamma) \times\left[-\alpha_{n}, \alpha_{n}\right]$. Recall from our choice of $\epsilon$ that the diameter of $H_{n}\left(\{\theta\} \times\left[-\alpha_{n}, \alpha_{n}\right]\right)$ is at least $1 / 4+\epsilon$. Thus we can find $z_{1}, z_{2} \in\{\theta\} \times\left[-\alpha_{n}, \alpha_{n}\right]$ such that $d\left(H_{n}\left(z_{1}\right), H_{n}\left(z_{2}\right)\right) \geqslant 1 / 4+\epsilon$. But then there exist $z_{1}^{\prime}, z_{2}^{\prime} \in V(\gamma)$ such that $d\left(z_{i}, z_{i}^{\prime}\right)<3 \delta$, and our choice of $\delta$ implies that $d\left(H_{n}\left(z_{i}\right), H_{n}\left(z_{i}^{\prime}\right)\right)<\epsilon / 2$ for $i \in\{1,2\}$. Thus $d\left(H_{n}\left(z_{1}^{\prime}\right), H_{n}\left(z_{2}^{\prime}\right)\right)>1 / 4$, and it follows that $H_{n}(V(\gamma))$ has diameter greater than $1 / 4$. Since $V(\gamma)=h_{n+1}\left(\{\theta\} \times\left[-\alpha_{n+1}, \alpha_{n+1}\right]\right)$, we see that property $(2)$ holds for $k=n+1$.

To verify property $\sqrt{(6)}$ for $k=n+1$, note that, using the properties of $V$ and $T$ and the fact that $\delta<1 / q_{n}$,

$$
d\left(\pi_{1}\left(h_{n+1}(z), \pi(z)\right)=d\left(\pi_{1}(V(T(z))), \pi_{1}(z)\right) \leqslant \delta+2 / q_{n}<3 / q_{n} .\right.
$$

The remaining properties are guaranteed by choosing $p_{n+1} / q_{n+1}$ close enough to $p_{n} / q_{n}$. Indeed, as noted at the beginning of the proof, properties (7) and (9) are guaranteed in this way, and property (3) will hold if $p_{n+1} / q_{n+1}$ is close to $p_{n} / q_{n}$ but not equal to it. Therefore there is an open interval $I$ containing $p_{n} / q_{n}$ such that properties (7) (8) and (3) hold as long as $p_{n+1} / q_{n+1} \in I \backslash\left\{p_{n} / q_{n}\right\}$.

By our induction assumption, property (9) holds for $f_{n}$, so that $\left\{f_{n}^{k}(z): 0 \leqslant k \leqslant M_{i}\right\}$ is $\epsilon_{i}$-dense in $\Lambda_{i}$ for each $z \in \Lambda_{n}$ and $1 \leqslant i \leqslant n-1$. However, this condition is $C^{0}$-open, meaning that it still holds if one replaces $f_{n}$ by a map which is $C^{0}$-close enough to $f_{n}$. Therefore, reducing the interval $I$ we may assume that whenever $p_{n+1} / q_{n+1} \in I \backslash\left\{p_{n} / q_{n}\right\}$, the set $\left\{f_{n+1}^{k}(z): 0 \leqslant k \leqslant M_{i+1}\right\}$ is $\epsilon_{i}$-dense in $\Lambda_{i}$ when $1 \leqslant i \leqslant n-1$ (noting that $\Lambda_{n+1} \subset \Lambda_{n}$ ). It remains to verify that there exists $M_{n+1}$ such that the latter also holds when $i=n+1$.

Choose an irrational $\theta \in I$ and note that by property (5) the set $H_{n+1}\left(\mathbb{T}^{1} \times\{\alpha\}\right)$ is $\epsilon_{n+1}$-dense in $\Lambda_{n}$ for any $\alpha \in\left[-\alpha_{n+1}, \alpha_{n+1}\right]$, so the map $g=H_{n+1} R_{\theta} H_{n+1}^{-1}$ has the property that every $g$-orbit of a point in $\Lambda_{n+1}$ is $\epsilon_{n+1}$-dense in $\Lambda_{n}$. By compactness, we may choose $M_{n+1}$ such that $\left\{g^{k}(z): 0 \leqslant k \leqslant M_{n+1}\right\}$ is $\epsilon_{n+1}$-dense in $\Lambda_{n}$ for any $z \in \Lambda_{n+1}$. Since this is an open condition, if $p_{n+1} / q_{n+1} \in I \backslash\left\{p_{n} / q_{n}\right\}$ is chosen close enough to $\theta$ we have that $\left\{f_{n+1}^{k}(z): 0 \leqslant k \leqslant M_{n+1}\right\}$ is $\epsilon_{n+1}$-dense in $\Lambda_{n}$, as required. Thus property (9) holds, completing the proof.

\section{References}

[AK70] D. Anosov and A. Katok, New examples in smooth ergodic theory. Ergodic diffeomorphisms, Trans. Mosc. Math. Soc. 23 (1970), 1-35. 
[Aus88] J. Auslander, Minimal flows and their extensions, Elsevier Science Ltd, 1988.

[Bel78] H. Bell, A fixed point theorem for plane homeomorphisms, Fund. Math. 100 (1978), no. 2, 119128.

[BG91] M. Barge and R. M. Gillette, Rotation and periodicity in plane separating continua, Ergodic Theory Dyn. Syst. 11 (1991), 619-631.

[BGM93] B. L. Brechner, M. D. Guay, and J. C. Mayer, The rotational dynamics of cofrontiers, Continuum theory and dynamical systems, Lecture Notes in Pure and Appl. Math., vol. 149, Dekker, New York, 1993, pp. 59-82.

[Bin51] R. H. Bing, Concerning hereditarily indecomposable continua., Pac. J. Math. 1 (1951), 43-51.

[BO] J. Boronski and P. Oprocha, Rotational chaos and strange attractors on the 2-torus, Preprint 2014.

[CL51a] M. L. Cartwright and J. E. Littlewood, Some fixed point theorems, Ann. of Math. (2) 54 (1951), 1-37.

[Cha73] J. J. Charatonik, On decompositions of continua, Fund. Math. 79 (1973), no. 2, $113-130$.

[Dav] P. Davalos, On annular maps of the torus and sublinear diffusion, To appear in Journal of the Institute of Mathematics of Jussieu, 2016.

[Dav86] R. J. Daverman, Decompositions of manifolds, Pure and Applied Mathematics, vol. 124, Academic Press Inc., Orlando, FL, 1986.

[Ell69] R. Ellis, Lectures on topological dynamics, WA Benjamin, 1969.

[FK04] B. Fayad and A. Katok, Constructions in elliptic dynamics, Ergodic Theory Dyn. Syst. 24 (2004), no. 5, 1477-1520.

[FS67] R. W. FitzGerald and P. M. Swingle, Core decomposition of continua, Fund. Math. 61 (1967), 33-50.

[FLC03] J. Franks and P. Le Calvez, Regions of instability for non-twist maps, Ergodic Theory Dyn. Syst. 23 (2003), no. 1, 111-141.

[GKT14] N. Guelman, A. Koropecki, and F. A. Tal, A characterization of annularity for area-preserving toral homeomorphisms, Math. Z. 276 (2014), no. 3-4, 673-689.

[Han82] M. Handel, A pathological area preserving $C^{\infty}$ diffeomorphism of the plane, Proc. Am. Math. Soc. 86 (1982), no. 1, 163-168.

[Her83] M. Herman, Une méthode pour minorer les exposants de Lyapunov et quelques exemples montrant le caractère local d'un théorème d'Arnold et de Moser sur le tore de dimension 2, Comment. Math. Helv. 58 (1983), 453-502.

[Her86]_ Construction of some curious diffeomorphisms of the Rieman sphere, J. Lond. Math. Soc. 34 (1986), 375-384.

[HY61] JG Hocking and GS Young, Topology, Addison Wesley, 1961.

[Jäg09a] T. Jäger, The concept of bounded mean motion for toral homeomorphisms, Dyn. Syst. 24 (2009), no. 3, 277-297.

[Jäg09b] Linearisation of conservative toral homeomorphisms, Invent. Math. 176 (2009), no. 3, 601-616.

[Jäg10]_ Periodic point free homeomorphisms of the open annulus - from skew products to non-fibred maps, Proc. Am. Math. Soc. 138 (2010), 1751-1764.

[JP] T. Jäger and A. Passeggi, On torus homeomorphisms semiconjugate to irrational rotations, Ergodic Theory Dyn. Syst. 35 (2015), no. 7, 2114-2137.

[KY94] J. A. Kennedy and J. A. Yorke, Pseudocircles in dynamical systems, Trans. Am. Math. Soc. 343 (1994), no. 1, 349-366.

[Kor10] A. Koropecki, Aperiodic invariant continua for surface homeomorphisms, Math. Zeitschrift 266 (2010), no. 1, 229-236.

[KLCN15] A. Koropecki, P. Le Calvez, and M. Nassiri, Prime ends rotation numbers and periodic points, Duke Math. J 164 (2015), no. 3, 403-472. 
[KN10] A. Koropecki and M. Nassiri, Transitivity of generic semigroups of areapreserving surface diffeomorphisms, Math. Z. 266 (2010), no. 3, 707-718.

[Kur27] C. Kuratowski, Théorie des continus irréductibles entre deux points II, Fund. Math. 10 (1927), 225-275.

[Kur28] _ Sur la structure des frontières communes 'a deux régions, Fund. Math. 12 (1928), 20-42.

[LC88] P. Le Calvez, Propriétés des attracteurs de Birkhoff., Ergodic Theory Dyn. Syst. 8 (1988), no. 2, 241-310.

[MMO01] A. O. Maner, J. C. Mayer, and L. G. Oversteegen, Cantor sets of arcs in decomposable local Siegel disk boundaries Topology Appl. 112 (2001), no. 3, 315336.

[New92] M.H.A. Newman, Elements of the topology of plane sets of points, Dover Publications, 1992.

[Rak77] Z. M. Rakowski, Monotone decompositions of continua, Fund. Math. 94 (1977), no. 2, 155-163.

[Tur12] M. Turpin, A pseudorotation with quadratic irrational rotation number, Proc. Amer. Math. Soc. 140 (2012), no. 1, 227233.

[Vee65] W. A. Veech, Almost automorphic functions on groups, Am. J. Math. 87 (1965), no. 3, 719-751.

[Vou74] E. J. Vought, Monotone decompositions of continua not separated by any subcontinua, Trans. Amer. Math. Soc. 192 (1974), 67-78.

[Wal91] R. Walker, Periodicity and Decomposability of Basin Boundaries with Irrational Maps on Prime Ends, Transactions of the A.M.S. 324 (1991), no. 1, 303-317.

[Why55] G. T. Whyburn, Analytic topology, American Mathematical Society. Colloquium publications, no. v. 28, pt. 2, American Mathematical Soc., 1955. 